\title{
ŁOJASIEWICZ EXPONENTS OF A CERTAIN ANALYTIC FUNCTIONS
}

\author{
MUTSUO OKA
}

\begin{abstract}
We consider the exponent of Łojasiewicz inequality $\|\partial f(\mathbf{z})\| \geq$ $c \mid f\left(\left.\mathbf{z}\right|^{\theta}\right.$ for two classes of analytic functions and we will give an explicit estimation for $\theta$. First we consider certain non-degenerate functions which is not convenient. In $\S 3.4$, we give an example of a polynomial for which $\theta_{0}(f)$ is not constant on the moduli space and in $\S 3.5$, we show that the behaviors of the Eojasiewicz exponents is not similar as the Milnor numbers by an example.

In the last section $(\S 4)$, we give also an estimation for product functions $f(\mathbf{z})=f_{1}(\mathbf{z}) \cdots f_{k}(\mathbf{z})$ associated to a family of a certain convenient non-degenerate complete intersection varieties. In either class, the singularity is not isolated. We will give explicit estimations of the Łojasiewicz exponent $\theta_{0}(f)$ using combinatorial data of the Newton boundary of $f$. We generalize this estimation for non-reduced function $g=f_{1}^{m_{1}} \cdots f_{k}^{m_{k}}$.
\end{abstract}

\section{HolOMORPHIC FUNCTIONS AND ŁOJASIEWICZ EXPONENTS}

Consider a germ of an analytic function $f(\mathbf{z})$ at the origin. There are two type of inequalities which are shown by S. Łojasiewicz [16, 17].

$$
\begin{aligned}
\|\partial f(\mathbf{z})\| & \geq c|f(\mathbf{z})|^{\theta}, c \neq 0,0 \leq \exists \theta<1, \forall \mathbf{z} \in U, \\
\|\partial f(\mathbf{z})\| & \geq c\|\mathbf{z}\|^{\eta}, c \neq 0, \exists \eta>0, \forall \mathbf{z} \in U
\end{aligned}
$$

where $U$ is a sufficiently small neighborhood of the origin. Here $\partial f(z)$ is the gradient vector $\left(\frac{\partial f}{\partial z_{1}}, \ldots, \frac{\partial f}{\partial z_{n}}\right)$. These equalities hold in a sufficiently small neighborhood of the origin. For the second inequality, $f(\mathbf{z})$ must have an isolated singularity at the origin. In our previous paper [20], we considered the exponent of the second Eojasiewicz inequality for a nondegenerate holomorphic function $f(\mathbf{z})$ (or a mixed function $f(\mathbf{z}, \overline{\mathbf{z}})$ ) with an isolated singularity at the origin. For further information about Łojasiewicz inequality (2), we refer [16, 17, 1, 2, 3, 5, 13, 20, 21, 22.

In this paper, we are interested in the exponent of the type (1) for a certain type of holomorphic functions which may have non-isolated singularities at the origin. This inequality has been originally studied by Łojasiewicz [16, 17] and then by many other authors. Most of the researches have been concentrated for the existence of the inequality in more general setting. For example, in the papers [15, 11, 12, the authors show the existence of

2000 Mathematics Subject Classification. 32S05.

Key words and phrases. Łojasiewicz exponent, inv-tame. 
Łojasiewicz inequality for the o-minimal situation. We study the exponent of the inequality (1) for a certain type of holomorphic functions which may have non-isolated singularities at the origin. They are either non-degenerate functions or the product of convenient non-degenerate polynomials associated to a non-degenerate complete intersection variety. The existence of Łojasiewicz inequality is well-known for holomorphic functions ([16, 17]). We are interested in the vanishing speed of the gradient vectors $\partial f(\mathbf{z})$ near the origin in comparison with that of the absolute value of $f$ when $\mathbf{z}$ goes to the origin. Thus we are mostly interested in the best possible $\theta$ which satisfies (1). This number is the infinimum of $\theta$ 's which satisfy (1) and we denote it by $\theta_{0}(f)$ hereafter.

Let $\mathbf{z}(t)$ be an analytic curve with $\mathbf{z}(0)=\mathbf{0}$ and $\mathbf{z}(t) \in \mathbb{C}^{n} \backslash f^{-1}(0)$ for $t \neq 0$. Then we compare the order of the both side of (1), after substituting $\mathbf{z}=\mathbf{z}(t)$ to get the inequality:

$$
\operatorname{ord}_{t}\|\partial f(\mathbf{z}(t))\| \leq \theta \times \operatorname{ord}_{t} f(\mathbf{z}(t))
$$

or equivalently

$$
\frac{\operatorname{ord}_{t}\|\partial f(\mathbf{z}(t))\|}{\operatorname{ord}_{t} f(\mathbf{z}(t))} \leq \theta .
$$

Using the Curve Selection Lemma $([14,8]), \theta_{0}(f)$ can be understood as the supremum of the left side ratios of the above inequality for all possible such curves $\mathbf{z}(t)$ and we call it the Eojasiewicz exponent of the function $f$ for the Łojasiewicz inequality (1).

\section{NON-DEGENERATE HYPERSURFACES}

2.1. Dual Newton diagram. We consider an analytic function (or a polynomial)

$$
f(\mathbf{z})=\sum_{\nu} c_{\nu} \mathbf{z}^{\nu}
$$

defined in the neighborhood of the origin. Recall that the Newton polyhedron $\Gamma_{+}(f)$ is the convex hull of the union $\bigcup_{\nu, c_{\nu} \neq 0}\left(\nu+\mathbb{R}_{+}^{n}\right)$. The Newton boundary $\Gamma(f)$ is defined by the union of compact faces of $\Gamma_{+}(f)$. Let $N^{+} \subset \mathbb{R}^{n}$ be the space of non-negative weight vectors. That is, a weight vector $P=\left(p_{1}, \ldots, p_{n}\right)$ is in $N^{+}$if and only if $p_{i} \geq 0$. It defines linear function $\ell_{P}$ of the Newton polyhedron $\Gamma_{+}(f)$ by $\ell_{P}(\nu)=\sum_{i=1}^{n} p_{i} \nu_{i}$ for $\nu \in \Gamma_{+}(f)$. Its minimal value is denoted by $d(P, f)$ and the face of $\Gamma_{+}(f)$, where this minimal value is taken, is denoted as $\Delta(P, f)$. If no ambiguity is likely, we simply denote it as $d(P)$ and $\Delta(P)$. We recall an equivalence relation in $N^{+}$which gives a polyhedral conical structure in $N^{+}$. Two weight vectors $P, Q$ are equivalent if and only if $\Delta(P)=\Delta(Q)$ and this equivalence gives a conical polyhedral subdivision of $N^{+}$which we call the dual Newton diagram and denote it as $\Gamma^{*}(f)$. The equivalence class of $P$ is denoted as $[P]$. 
2.2. Vanishing and non-vanishing weight vectors. For $I \subset\{1, \ldots, n\}$, we put $\mathbb{C}^{I}=\left\{\mathbf{z} \mid z_{j}=0, \forall j \notin I\right\}$. Thus $\mathbb{C}^{I} \subset \mathbb{C}^{n}$. The subspace $\mathbf{C}^{I}$ is called a vanishing coordinate subspace if $f^{I} \equiv 0$. Note that $f^{I}$ is the restriction of $f$ to $\mathbb{C}^{I}$. Consider a weight vector $P=\left(p_{1}, \ldots, p_{n}\right)$. Put $I(P):=\left\{i \mid p_{i}=0\right\}$. Assume that $I(P) \neq \emptyset$. A weight vector $P$ is called $a$ vanishing weight vector (respectively non-vanishing weight vector if $d(P)>0$ (resp. if $d(P)=0$ ). Thus $\mathbb{C}^{I(P)}$ is a vanishing coordinate subspace if $P$ is a vanishing weight vector. We denote the sets of strictly positive weight vectors (i.e. $I(P)=\emptyset$ ), vanishing weight vectors and non-vanishing weight vectors by $\mathcal{W}_{+}(f), \mathcal{W}_{v}(f)$ and $\mathcal{W}_{n v}(f)$ respectively. Hereafter we simply denote them as $\mathcal{W}_{+}, \mathcal{W}_{v}, \mathcal{W}_{n v}$, if no ambiguity is likely.

2.3. Convexity of the equivalence class. Let $P$ be a weight vector in $N^{+}$ and let $[P]$ the set of equivalent weight vectors. The equivalence class $[P]$ is the interior of a polyhedral convex cone in $N^{+}$and $\operatorname{dim}[P]=n-\operatorname{dim} \Delta(P)$. This follows from the obvious equality:

$$
\begin{aligned}
& \Delta(P) \cap \Delta(Q) \neq \emptyset \Longrightarrow \\
& \Delta((1-t) P+t Q)=\Delta(P) \cap \Delta(Q), \quad 0<t<1 .
\end{aligned}
$$

Put $\operatorname{LS}(P, Q):=\{(1-t) P+t Q \mid 0 \leq t \leq 1\}$ and we call $\operatorname{LS}(P, Q)$ the line segment with ends $P, Q$. Consider the closure $\overline{[P]}$ of $[P]$ in the Euclidean topology. Then $Q \in \overline{[P]}$ if and only if $\Delta(Q) \supset \Delta(P)$ and $\overline{[P]}$ is also a closed polyhedral convex cone. We say $Q$ is on the boundary of $[P]$ if $[Q] \subset \overline{[P]} \backslash[P]$ and denote as $Q \succ P$. Note that $Q \succ P$ if and only if $\Delta(Q) \supsetneq \Delta(P)$. We visualize $\Gamma^{*}(f)$ by cutting $N^{+}$by some transversal hyperplane $\Pi$ to the cone, say $\Pi: \nu_{1}+\cdots+\nu_{n}=1$ and we see the silhouette. See Figure 2 . In the figure, the dimension of the equivalence class is one less. Let $P$ a weight vector. We say that $P$ is a vertex of the dual Newton diagram $\Gamma^{*}(f)$ if and only if $\operatorname{dim} \Delta(P)=n-1$ or equivalently $\operatorname{dim}[P]=1$.

Definition 1. $f$ is called $k$-convenient if $f^{I} \not \equiv 0$ for any $I \subset\{1, \ldots, n\}$ with $|I| \geq n-k$, We say for simplicity $f$ is convenient if $f$ is $(n-1)$-convenient $(18])$.

2.4. Face function, non-degeneracy and tameness. Let $\Xi$ be a face of $\Gamma_{+}(f)$. The face function of $\Xi$ is defined by $f_{\Xi}(\mathbf{z}):=\sum_{\nu \in \Xi} c_{\nu} \mathbf{z}^{\nu}$. For a weight vector $P$, we define $f_{P}(\mathbf{z}):=f_{\Delta(P)}(\mathbf{z})$. If $P \in \mathcal{W}_{+} \cup \mathcal{W}_{v}$, then $d(P)>0$ and $f_{P}$ is a weighted homogeneous polynomial of $\mathbf{z}_{J}, J=I(P)^{c}$, of degree $d(P)$ with respect to the weight $P$. Here $I(P)^{c}=\{1, \ldots, n\} \backslash I(P)$ and $\mathbf{z}_{I}=\left(z_{i} \mid i \in I\right\}$. We recall that $f$ is non-degenerate if the mapping $f_{P}: \mathbb{C}^{* n} \rightarrow \mathbb{C}$ has no critical point for any $P \in \mathcal{W}_{+}$. Recall that $\mathbb{C}^{* n}:=$ $\left\{\mathbf{z} \in \mathbb{C}^{n} \mid z_{i} \neq 0,1 \leq i \leq n\right\}$.

We say that $f$ is locally tame (or strongly locally tame) if for any weight vector $P \in \mathcal{W}_{v}$, the face function $f_{P}: \mathbb{C}^{* I(P)^{c}} \rightarrow \mathbb{C}$ has no critical point as a function of variables $\mathbf{z}_{I(P)^{c}}$ for any sufficiently small (resp. for any) 
$\mathbf{z}_{I} \in \mathbb{C}^{* I(P)}$ fixed $([7])$. Here $\mathbb{C}^{* I}=\left\{\mathbf{z} \in \mathbb{C}^{n} \mid z_{j}=0\right.$, iff $\left.j \notin I\right\}$. For $I=\{1, \ldots, n\}$, we write simply $\mathbb{C}^{* n}$ instead of $\mathbb{C}^{* I}$.

Definition 2. Let $\operatorname{Var}(P)=\left\{j \mid \frac{\partial f_{P}}{\partial z_{j}} \neq 0\right\}$. That is, $j \in \operatorname{Var}(\mathrm{P})$ if and only if $z_{j}$ appears in a monomial of $f_{P}(\mathbf{z})$. We call $\operatorname{Var}(P)$ the variables of $P$ or of $f_{P}$. Let $\widetilde{I}(P):=\bigcup\left\{I(Q) \mid, Q \succ P, Q \in \mathcal{W}_{v}\right\}$ and we put $\widetilde{\operatorname{Var}}(P):=$ $\operatorname{Var}(P) \backslash \widetilde{I}(P)$. We call $\left\{z_{j} \mid j \in \widehat{\operatorname{Var}}(P)\right\}$ invulnerable variables for $P$. Note that $\widetilde{I}(P) \supset \widetilde{I}(Q)$ if $Q \succ P$. We introduce a stronger tameness: $f$ is strongly inv-tame for $P$ if $\widehat{\operatorname{Var}}(P)$ is not empty and $f_{P}: \mathbb{C}^{* n} \rightarrow \mathbb{C}$ has no critical point as a polynomial of the invulnerable variables $\left\{z_{j}, \mid j \in \widetilde{\operatorname{Var}}(P)\right\}$ for any $\mathbf{z}_{\widetilde{I}(P)} \in \mathbb{C}^{* \widetilde{I}(P)}$ fixed. We say $f$ is strongly inv-tame if any weight vector $P \in \mathcal{W}_{+} \cup \mathcal{W}_{v}$ with $\operatorname{dim} \Delta(P) \geq 1, f$ is strongly inv-tame for $P$.

For example, consider a weight vector $D$ on the open interval $\operatorname{Int}\left(\mathrm{RE}_{3}\right)$ in $f_{1}(\mathbf{z})$ (Figure 2). Then $R, E_{3} \succ D$. But $E_{3} \in \mathcal{W}_{n v}$. Thus $\widetilde{I}(D)=\{1\}$ and $f_{1 D}=z_{1}^{5} z_{2}^{2}$ and we see $f_{1}$ is strongly inv-tame for $D$.

Remark 3. Assume that $f$ is $(n-2)$-convenient. Take $P \in \mathcal{W}_{+} \cup \mathcal{W}_{v}$ with $\operatorname{dim} \Delta(P) \geq 1$. Assume that $Q \in \overline{[P]}$ and $Q \in \mathcal{W}_{v}$. Then $\sharp I(Q)=1$. (If $\sharp(I(Q))=2, Q \in \mathcal{W}_{n v}$.) If $P \in \mathcal{W}_{v}, I(Q)=I(P)$. Thus it is easy to check if $P$ is strongly inv-tame or not.

2.5. Dimension of $[P]$. We recall the following relation of the dimension of the equivalence class $[P]$ and the dimension of $\Delta(P)$ :

$$
\operatorname{dim}[P]=n-\operatorname{dim} \Delta(P) .
$$

Suppose that $I(P) \neq \emptyset$ and put $I:=I(P)$. Consider $f$ as a polynomial $f(\mathbf{z}) \in K\left[\mathbf{z}_{I^{c}}\right]$ with the coefficient ring $K:=\mathbb{C}\left[\mathbf{z}_{I}\right]$. We use the notation ${ }^{K} f$ when we consider $f$ as a polynomial in $K\left[\mathbf{z}_{I^{c}}\right]$. Note that $\operatorname{dim} \Delta_{c}(P)=$ $\operatorname{dim} \Delta\left(P_{I^{c}},{ }^{K} f\right)$ and

$$
\operatorname{dim} \Delta(P)=\operatorname{dim} \Delta_{c}(P)+\sharp I .
$$

where $\sharp I$ is the cardinality of $I$. Here $\Delta_{c}(P)$ is defined by $\Delta(P) \cap \Gamma(f)$.

2.6. Normalized weight vector. Take a weight vector $P=\left(p_{1}, \ldots, p_{n}\right) \in$ $\mathcal{W}_{+} \cup \mathcal{W}_{v}$. Then $d(P)>0$. We consider the rational weight vector $\hat{P}=$ $\left(\hat{p}_{1}, \ldots, \hat{p}_{n}\right)$ which is defined by $\hat{P}=P / d(P)$. That is, $\hat{p}_{i}=p_{i} / d(P), i=$ $1, \ldots, n$. It is clear that $P$ and $\hat{P}$ are equivalent and $d(\hat{P})=1$. We use this notation throughout the paper and we call $\hat{P}$ the normalized weight vector of $P$. If $d(P)=0, P$ does not have any normalized form. Using the normalized weight vector, each monomial in $f_{\hat{P}}(\mathbf{z})$ has weight 1 . For given two weight vectors $P$ and $Q$ with $d(P), d(Q)>0$ and $\Delta(P, Q):=\Delta(P) \cap \Delta(Q) \neq \emptyset$, consider the line segment $\operatorname{LS}(P, Q)=\left\{\hat{P}_{t} \mid 0 \leq t \leq 1\right\}$ where $\hat{P}_{t}:=(1-$ $t) \hat{P}+t \hat{Q}$. The $i$-component $\hat{p}_{t i}$ of $\hat{P}_{t}$ is given as $\hat{p}_{t i}=(1-t) \hat{p}_{i}+t \hat{q}_{i}$ and it is monotone (either increasing or constant or decreasing) function in $t$ for any $1 \leq i \leq n$. In particular, 
Proposition 4. There is a canonical inequality: $\hat{p}_{t i} \geq \min \left\{\hat{p}_{i}, \hat{q}_{i}\right\}$.

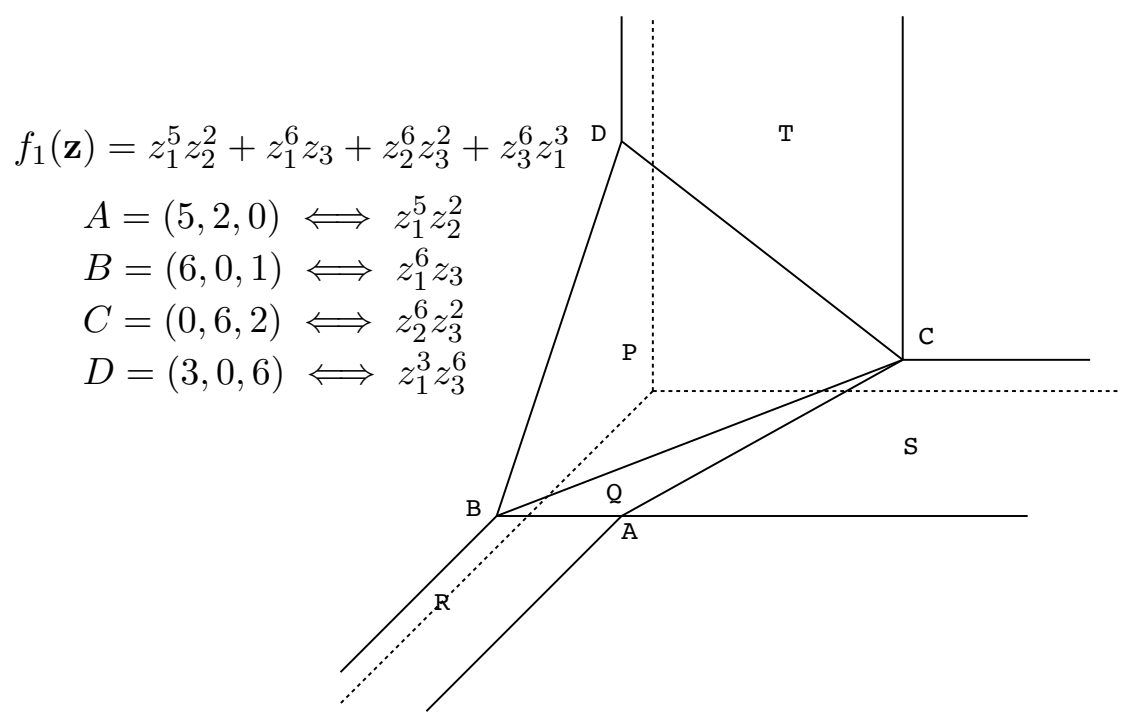

FiguRE 1. Newton boundary of $f_{1}$

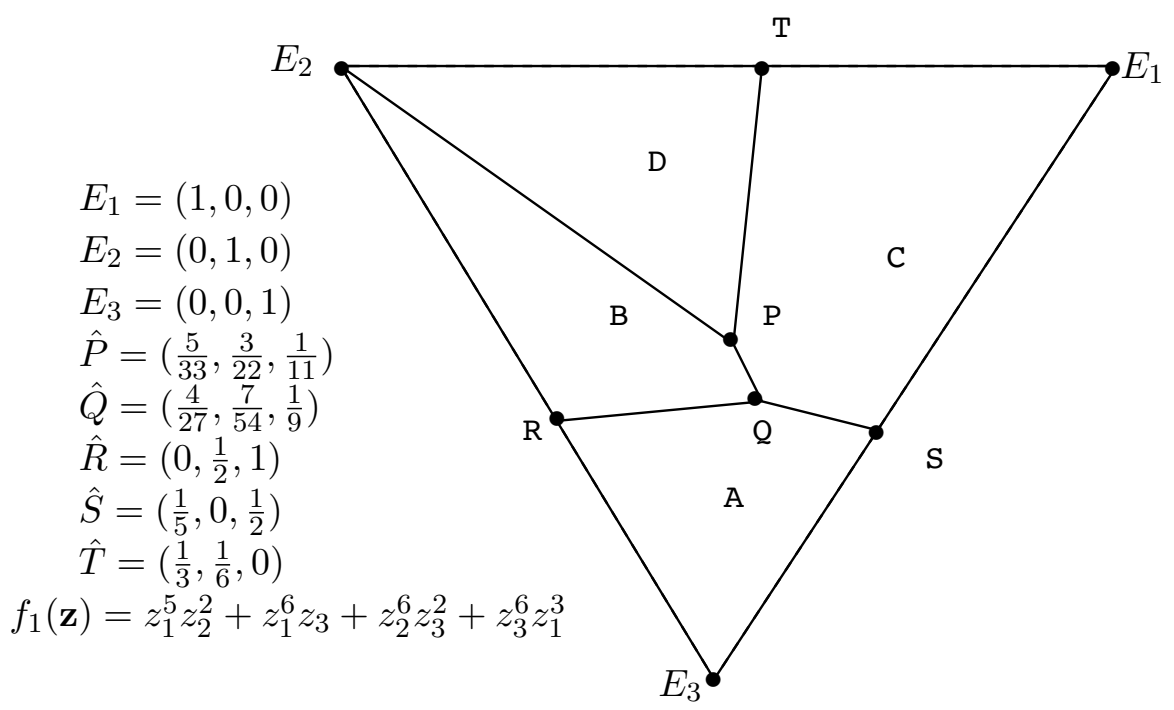

Figure 2. $\Gamma^{*}\left(f_{1}\right)$ 


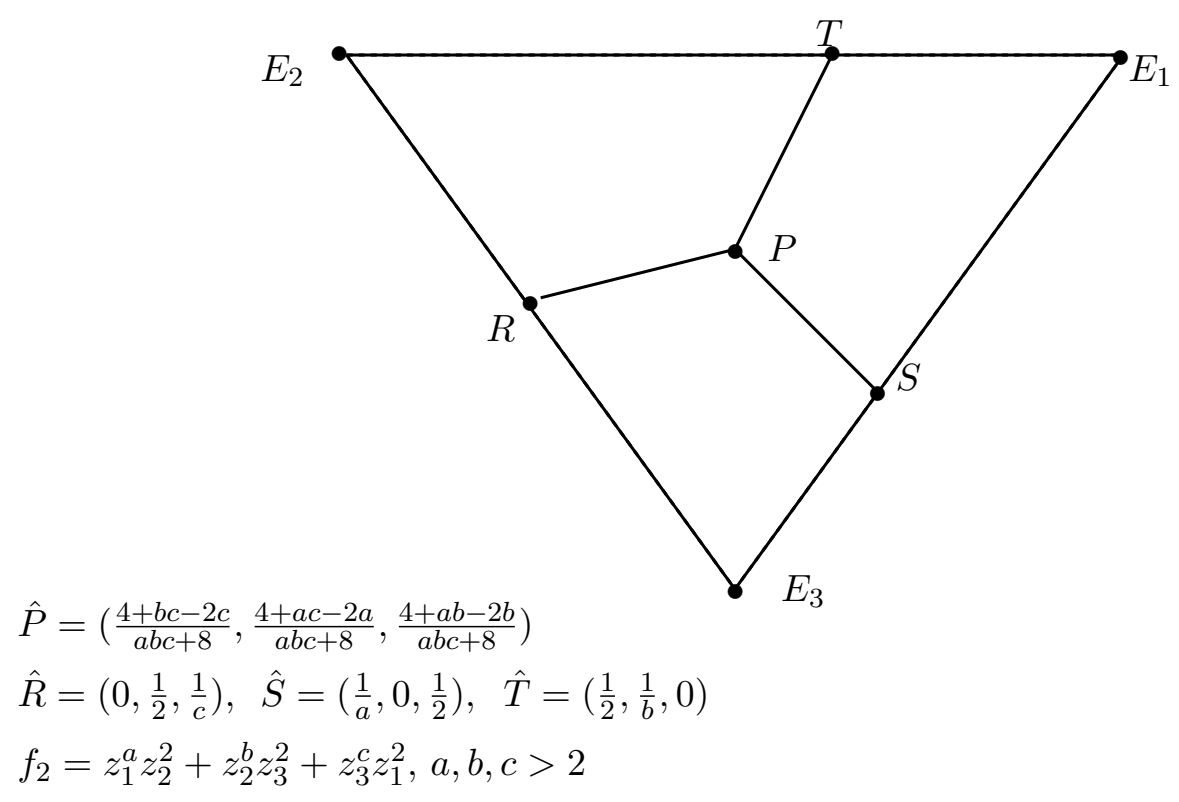

Figure 3. $\quad \Gamma^{*}\left(f_{2}\right)$

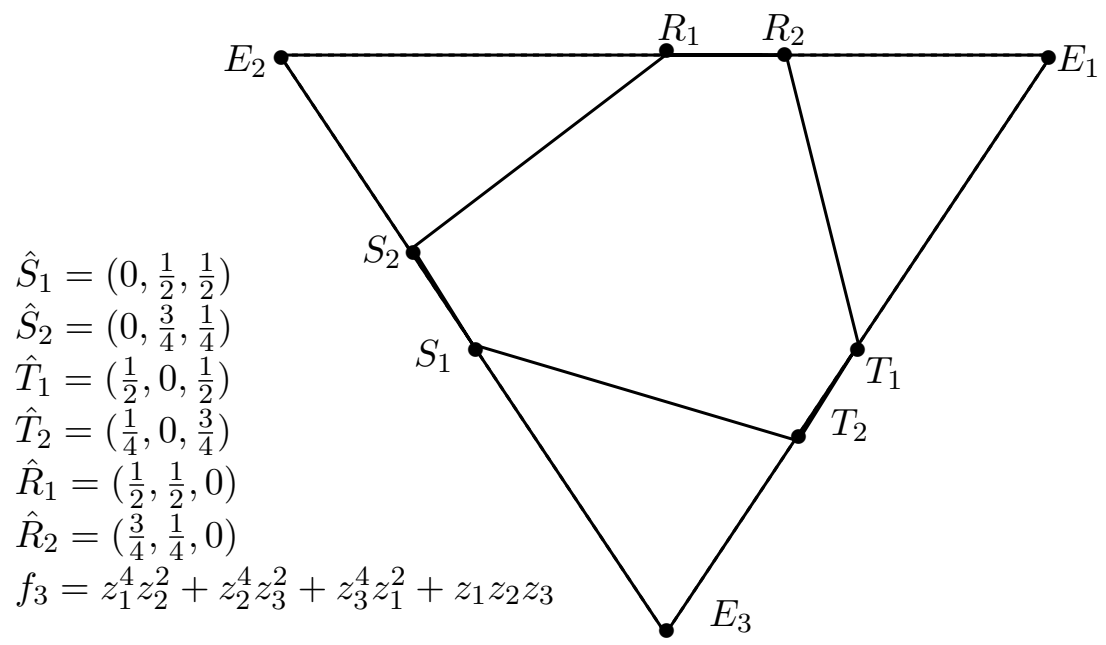

FiguRE 4. $\Gamma^{*}\left(f_{3}\right)$

2.7. Examples. In the following, polynomials in Example 1, Example 2 and Example 3 are all non-degenerate and strongly inv-tame. 
Example 5. Consider $f_{1}(\mathbf{z})=z_{1}^{4} z_{2}^{2}+z_{1}^{6} z_{3}+z_{2}^{6} z_{3}^{2}+z_{1}^{3} z_{3}^{6}$. Figure 1 and Figure 2 show the Newton boundary and the dual Newton diagram of $f_{1}$ respectively. In Figure 2, we see 8 equivalence clasees which correspond to the vertices $P, Q \in \mathcal{W}_{+}, R, S, T \in \mathcal{W}_{v}$ and $E_{1}, E_{2}, E_{3} \in \mathcal{W}_{n v}, 11$ equivalence classes corresponding to the edges and 4 equivalence classes which corresponds to the interiors of four polyhedra in Figure 2. Here $E_{1}, E_{2}, E_{3}$ are the standard basis of $\mathbb{R}^{3}$.

Example 6. (Weighted homogeneous case) Consider the weighted homogeneous polynomial $f_{2}(\mathbf{z})=z_{1}^{a} z_{2}^{2}+z_{2}^{b} z_{3}^{2}+z_{3}^{c} z_{1}^{2}$ with $a, b, c>2$. The dual Newton diagram is given in Figure 3.

Example 7. Consider the polynomial $f_{3}(\mathbf{z})=z_{1}^{4} z_{2}^{2}+z_{2}^{4} z_{3}^{2}+z_{3}^{4} z_{1}^{2}+z_{1} z_{2} z_{3}$. This polynomial is special in the sense that its Newton boundary $\Gamma(f)$ does not have any compact 2-dimensional face. See Figure 4.

2.8. Ratio maps for curves in the coordinate subspaces. Assume that $\mathbb{C}^{I}$ is a non-vanishing coordinate subspace. Let $\mathcal{P}_{I}$ be the set of analytic curves $C: \mathbf{z}=\mathbf{z}(t), 0 \leq t \leq 1$ such that its image is in $\mathbb{C}^{I} \subset \mathbb{C}^{n}, \mathbf{z}(0)=\mathbf{0}$ and $\mathbf{z}(t) \in \mathbb{C}^{* I} \backslash V\left(f^{I}\right)$ for $t \neq 0$. For a curve $\mathbf{z}(t) \in \mathcal{P}_{I}$, we consider the ratio map

$$
\theta: \mathcal{P}_{I} \rightarrow[0,1), \quad \theta(C)=\theta(\mathbf{z}(t))=\frac{\operatorname{ord}_{t}\|\partial f(\mathbf{z}(t))\|}{\operatorname{ord}_{t} f(\mathbf{z}(t))}
$$

Note that $\|\partial f(\mathbf{z}(t))\|$ is measured in $\mathbb{C}^{n}$. Consider a modified curve $\widetilde{\mathbf{z}}(t)$ defined as $\widetilde{z}_{i}(t)=z_{i}(t)$ for $i \in I$ and $=t^{N}$ for $i \notin I$. Note that $\widetilde{\mathbf{z}}(t) \in \mathbb{C}^{* n}$ for $t \neq 0$. Let $\widetilde{P}=\left(\widetilde{p}_{i}, \ldots, \widetilde{p}_{n}\right)$ be the weight vector of $\widetilde{\mathbf{z}}(t)$. Thus $\widetilde{p}_{i}=p_{i}$ for $i \in I$ and $\widetilde{p}_{i}=N$ for $i \notin I$.

Proposition 8. Taking $N$ sufficiently large we have that

$$
\begin{aligned}
& \operatorname{ord}_{t} \partial f(\widetilde{\mathbf{z}}(t))=\operatorname{ord}_{t} \partial f(\mathbf{z}(t)) \leq \operatorname{ord}_{t} \partial f^{I}(\mathbf{z}(t)), \\
& \operatorname{ord}_{t} f(\mathbf{z}(t))=\operatorname{ord}_{t} f(\widetilde{\mathbf{z}}(t)) .
\end{aligned}
$$

Thus we have the equality:

$$
\frac{\operatorname{ord}_{t} \partial f(\mathbf{z}(t))}{\operatorname{ord}_{t} f(\mathbf{z}(t))}=\frac{\operatorname{ord}_{t} \partial f(\widetilde{\mathbf{z}}(t))}{\operatorname{ord}_{t} f(\widetilde{\mathbf{z}}(t))} .
$$

Proof. First observe that the difference

$$
f(\mathbf{z})-f\left(\mathbf{z}_{I}\right), \frac{\partial f}{\partial z_{i}}(\mathbf{z})-\frac{\partial f}{\partial z_{i}}\left(\mathbf{z}_{I}\right) \equiv 0 \quad \bmod \left(z_{j}\right)_{j \notin I} .
$$

Here $\left(z_{j}\right)_{j \notin I}$ is the ideal generated by $z_{j}, j \notin I$. Therefore taking $N$ sufficiently large we may assume that (i) $\Delta(\widetilde{P}, f)=\Delta\left(P, f^{I}\right)$ and

$$
\text { (ii) } \operatorname{ord}_{t}\left(\frac{\partial f}{\partial z_{j}}(\mathbf{z}(t))-\frac{\partial f}{\partial z_{j}}(\widetilde{\mathbf{z}}(t))\right) \geq N
$$

and (iii) $\operatorname{ord}_{t} f(\mathbf{z}(t))=\operatorname{ord}_{t} f(\widetilde{\mathbf{z}}(t))$. Then the assertion follows immediately. 
Thus we have

Corollary 9. $\theta(\mathbf{z}(t))=\theta(\widetilde{\mathbf{z}}(t))$.

Therefore for the estimation of $\theta_{0}(f)$, it is enough to consider the case $I=\{1, \ldots, n\}$, i.e. $\mathbf{z}(t) \in \mathbb{C}^{* n}$ for $t \neq 0$.

Lemma 10. Consider a weight vector $P \in \mathcal{W}_{+}$and assume that $f_{P}(\mathbf{z})=$ $c \mathbf{z}^{\nu}, c \neq 0$. Consider a normalized weight $\hat{P}$. Put $\hat{p}_{\max }:=\max \left\{\hat{p}_{i} \mid i \in\right.$ $\operatorname{Var}(\mathrm{P})\}$ and $|\nu|:=\sum_{i \in \operatorname{Var}(\mathrm{P})} \nu_{i}$. Then $\hat{p}_{\max } \geq \frac{1}{|\nu|}$.

Proof. The assertion is immediate from the equality

$$
1=\sum_{i \in \operatorname{Var}(\mathrm{P})} \hat{p}_{i} \nu_{i} \leq \hat{p}_{\max }|\nu|
$$

Corollary 11. Consider a curve $C$ parametrized as in Lemma 10. Then

$$
\theta(C)=1-\hat{p}_{\max } \leq 1-\frac{1}{|\nu|} .
$$

The assertion follows from the observation: $\frac{\partial f_{P}}{\partial z_{j}}(\mathbf{a}) \neq 0$ for any $j \in \operatorname{Var}(\mathrm{P})$ and $\mathbf{a} \in \mathbb{C}^{* n}$.

2.9. Ratio maps for weight vectors. We assume that $f$ is strongly invtame. Let $\mathcal{W}_{+}$be the set of strictly positive weight vectors and let $\mathcal{W}_{v}$ be the subset of positive weight vectors such that $I(P) \neq \emptyset$ and $d(P)>0$. Let $C=\{z=\mathbf{z}(t)\} \in \mathcal{P}$ and consider the Taylor expansion

$$
z_{i}(t)=a_{i} t^{p_{i}}+\text { (higher terms), } a_{i} \neq 0,1 \leq i \leq n .
$$

We consider the weight map $w t: \mathcal{P} \rightarrow \mathcal{W}$ by wt $(\mathbf{z}(t))=P$ where $P=$ $\left(p_{1}, \ldots, p_{n}\right)$. We want to estimate the ration $\theta(\mathbf{z}(t))$ using the weight vector $\operatorname{wt}(\mathbf{z}(t))$.

Definition 12. We define the ratio maps for weight vector $P \in \mathcal{W}_{+} \cup \mathcal{W}_{v}$ as follows. First we put

$$
\begin{aligned}
& \hat{p}_{\text {min }}:=\min \left\{\hat{p}_{j} \mid j \in \operatorname{Var}(\mathrm{P})\right\}, \\
& \hat{p}_{\text {min }}^{\prime}:=\min \left\{\hat{p}_{j} \mid j \in \widetilde{\operatorname{Var}}(P)\right\} .
\end{aligned}
$$

We define

$$
\begin{aligned}
& \theta_{i}(P)=1-\hat{p}_{i}, \quad i \in \widetilde{\operatorname{Var}}(P), \operatorname{dim} \Delta(P) \geq 1, \\
& \theta(P)=1-\hat{p}_{\text {min }}, \\
& \theta(P)^{\prime}= \begin{cases}1-\hat{p}_{\text {min }}^{\prime}, & \operatorname{dim} \Delta(P) \geq 1, \\
1-\frac{1}{|\nu|}, & \operatorname{dim} \Delta(P)=0, f_{P}(\mathbf{z})=c_{\nu} \mathbf{z}^{\nu} .\end{cases}
\end{aligned}
$$

Here $P=\left(p_{1}, \ldots, p_{n}\right)$ and $\hat{P}=\left(\hat{p}_{1}, \ldots, \hat{p}_{n}\right)$ is the normalized weight vector of $P$. 
As a special case, we have

Proposition 13. Assume that $\operatorname{dim} \Delta(P)=n-1$. Then $I(P)=\widetilde{I}(P)$ and $\hat{p}_{\text {min }}^{\prime}=\min \left\{\hat{p}_{j} \mid j \in \operatorname{Var}(\mathrm{P}) \backslash \mathrm{I}(\mathrm{P})\right\}$. In particular, $\hat{p}_{\text {min }}^{\prime}=\hat{p}_{\text {min }}$ if $P \in \mathcal{W}_{+}$.

2.10. Admissible line segments. We assume that $f$ is strongly inv-tame and non-degenerate. Consider a weight vector $R \in \mathcal{W}_{+} \cup \mathcal{W}_{v}$ with $\operatorname{dim} \Delta(R) \geq$ 1. Consider a line segment $\operatorname{LS}(P, Q)$ passing through $R$ with two weight vectors $P, Q$ on the boundary of $[R]$. Recall that $\operatorname{LS}(P, Q)=\left\{P_{s}=(1-s) P+\right.$ $s Q \mid 0 \leq s \leq 1\}$. By the assumption, $R=P_{s_{0}}, 0<\exists s_{0}<1$. We devide the situation into three cases depending the end points $P, Q$.

2.10.1. Strictly positive line segment. Let $R$ be as above. We say that the boundary of $[R]$ is strictly positive if the closure of the equivalence class $\overline{[R]}$ contains only strictly positive weight vectors. Thus $P, Q \in \mathcal{W}_{+}$. We use the line segment expression using the normalized vectors $\hat{P}_{s}=(1-s) \hat{P}+s \hat{Q}$. Then $R=P_{s_{0}^{\prime}}$ for some $s_{0}^{\prime}$. As the normalized weight vector $\hat{P}_{s}$ is given as $\hat{p}_{s j}=(1-s) \hat{p}_{j}+s \hat{q}_{j}$ is a monotone linear function in $s$, it is easy to see that

$$
\begin{aligned}
\theta_{j}(\hat{R}) & \leq \max \left\{\theta_{j}(\hat{P}), \theta_{j}(\hat{Q})\right\}, \quad j \in \operatorname{Var}(\hat{R}), \\
\theta(\hat{R})^{\prime} & \leq \max \{\theta(\hat{P}), \theta(\hat{Q})\} .
\end{aligned}
$$

Note that in this case, $\widetilde{I}(R)=\emptyset$ and $\theta(\hat{R})^{\prime}=\theta(\hat{R}), \theta(\hat{P})^{\prime}=\theta(\hat{P})$ and $\theta(\hat{Q})^{\prime}=\theta(\hat{Q})$, as $\widetilde{I}(R)=\emptyset$. For example, take $R$ on the line segment $\mathrm{LS}(P, Q)$ in Example 1 ( Figure 2).

Remark 14. Note that $\operatorname{Cone}(\operatorname{LS}(P, Q))=\operatorname{Cone}(\operatorname{LS}(\hat{P}, \hat{Q}))$, though $(1-$ $t) \hat{P}+t \hat{Q}$ is not necessarily the normalized vector of $(1-t) P+t Q$. Here for a subset $K \subset N^{+}$, we put $\operatorname{Cone}(K):=\{r P \mid P \in K, r>0\}$.

2.10.2. Vanishing line segment. We say that $\operatorname{LS}(P, Q)$ is a vanishing line segment if $P, Q$ are in $\mathcal{W}_{+} \cup \mathcal{W}_{v}$ and at least one of $P$ or $Q$ is in $\mathcal{W}_{v}$.

Lemma 15. Assume that $\operatorname{LS}(P, Q)$ is a vanishing line segment. We assume that $P \in \mathcal{W}_{v}, Q \in \mathcal{W}_{+} \cup \mathcal{W}_{v}$. Consider the family of the normalized weight vectors $\hat{P}_{s}$ for the line segment $\operatorname{LS}(P, Q)$ which is defined as $\hat{P}_{s}=(1-s) \hat{P}+$ $s \hat{Q}$ for $0 \leq s \leq 1$ and $\hat{R}=\hat{P}_{s_{0}^{\prime}}\left(0<\exists s_{0}^{\prime}<1\right)$. Then

$$
\begin{aligned}
& \theta_{j}(\hat{R}) \leq \max \left\{\theta_{j}(\hat{P}), \theta_{j}(\hat{Q})\right\}, \quad j \in \widetilde{\operatorname{Var}}(R), \\
& \theta(\hat{R})^{\prime} \leq \max \left\{\theta(\hat{P})^{\prime}, \theta(\hat{Q})^{\prime}\right\} .
\end{aligned}
$$

Proof. By the strong inv-tameness, $\widetilde{\operatorname{Var}}(R) \neq \emptyset$ and there exists a $j \in \widetilde{\operatorname{Var}}(R)$ so that $\frac{\partial f_{\hat{P}_{s_{0}^{\prime}}}}{\partial z_{j}}(\mathbf{a}) \neq 0$. This implies $\theta(\hat{R})^{\prime} \leq 1-\hat{p}_{s_{0}^{\prime}, j}$. The assertion follows from the monotonicity of $\hat{p}_{t j}$. 
2.10.3. Non-vanishing line segment. A line segment $\operatorname{LS}(P, Q)$ is called nonvanishing line segment if one of $P, Q$ is a non-vanishing weight vector. Assume that $P \in \mathcal{W}_{n v}$ and $Q \in \mathcal{W}_{+} \cup \mathcal{W}_{v}$ so that $\Delta(Q) \cap \Delta(P) \supset \Delta(R)$. Recall that $\operatorname{LS}(P, Q)$ is defined by $\left\{P_{s} \mid 0 \leq s \leq 1\right\}$ where $P_{s}:=(1-s) P+s Q$ and $R=\left(1-s_{0}\right) P+s_{0} Q$ as before. The normalized weight vectors of this family is written as $\hat{P}_{\tau}:=\tau P+\hat{Q}$ with $\tau=\frac{1-s}{s}$ for $s \neq 0$. In this parameter $\tau, 0 \leq \tau<\infty$ and $\hat{R}=\hat{P}_{\tau_{0}}, \exists \tau_{0}>0$. Note that $\hat{p}_{\tau, j}$ is monotone increasing (or constant) in $\tau$ for any $j$. That is $\hat{p}_{\tau, j} \geq \hat{p}_{0, j}=\hat{q}_{j}$.

Lemma 16. We have the inequality:

$$
\begin{aligned}
& \theta_{j}(\hat{R}) \leq \theta_{j}(\hat{Q}), \quad j \in \widetilde{\operatorname{Var}}(R), \\
& \theta(\hat{R})^{\prime} \leq \theta(\hat{Q})^{\prime} .
\end{aligned}
$$

Remark 17. We do not need to consider the case where $P, Q$ are both nonvanishing, as $R$ is assumed to be in $\mathcal{W}_{+} \cup \mathcal{W}_{v}$. In the inductive argument on $\operatorname{dim}[R]$, if the line segment is as in Lemma 16, we continue to work only for $Q$.

\section{Main RESUlt ON NON-DEGEnERATE FUnCTIONS}

3.1. Convenient case. Assume that $f(\mathbf{z})=\sum_{\nu} c_{\nu} \mathbf{z}^{\nu}$ is a convenient nondegenerate analytic function in the sense of Kouchnirenko [10] and let $b_{j}$ be the point $\Gamma(f) \cap\{\mathrm{j}$-th coordinate axis $\}$. Then $V(f)$ has an isolated singularity at the origin (Theorem (3.4), [18], Corollary 20, [19]). Consider an analytic curve $\mathbf{z}(t), 0 \leq t \leq 1$ as in the previous section. Namely $\mathbf{z}(0)=\mathbf{0}$ and $\mathbf{z}(t) \in \mathbb{C}^{n} \backslash f^{-1}(0)$ for $t \neq 0$. We first assume that $\mathbf{z}(t) \in \mathbb{C}^{* n}$ for $t \neq 0$. Assume that $\mathbf{z}(t)$ has the following Taylor expansion:

$$
\begin{aligned}
z_{j}(t) & =a_{j} t^{p_{j}}+(\text { higher terms }), \quad a_{j} \neq 0, j=1, \ldots, n, \\
\frac{\partial f}{\partial z_{j}}(\mathbf{z}(t)) & =\frac{\partial f_{P}}{\partial z_{j}}(\mathbf{a}) t^{d(P)-p_{j}}+(\text { higher terms }), \\
f(\mathbf{z}(t)) & =b t^{d^{\prime}}+(\text { higher terms }), b \neq 0, d^{\prime} \geq d(P) .
\end{aligned}
$$

where $P=\left(p_{1}, \ldots, p_{n}\right)$, a $=\left(a_{1}, \ldots, a_{n}\right)$. We use the same notation as in [18]. Put $p_{\min }=\min \left\{p_{j} \mid j \in \operatorname{Var}(\mathrm{P})\right\}$. Choose index $1 \leq \alpha \leq n$ so that $p_{\alpha}=p_{\min }$. Note that $\alpha$ may not unique but we fix it. If $f(\mathbf{z})$ is non-degenerate, then there exists $j_{0}$ so that $\frac{\partial f_{P}}{\partial z_{j_{0}}}(\mathbf{a}) \neq 0$. Thus

$$
\begin{gathered}
\operatorname{ord}_{t} \partial f(\mathbf{z}(t)) \leq d(P)-p_{j_{0}} \leq d(P)-p_{\text {min }}, \\
\frac{\operatorname{ord}_{t} \partial f(\mathbf{z}(t))}{\operatorname{ord}_{t} f(\mathbf{z}(t))} \leq \frac{d(P)-p_{\text {min }}}{d^{\prime}} \leq \frac{d(P)-p_{\text {min }}}{d(P)} .
\end{gathered}
$$

Use the normalized weight $\hat{P}=\left(\hat{p}_{1}, \ldots, \hat{p}_{n}\right)$ where $\hat{p}_{j}:=p_{j} / d(P)$. The right side of $(7)$ is equal to $1-1 / b_{\alpha}^{\prime}$ where $b_{\alpha}^{\prime}$ is the $\alpha$-coordinate so that $\hat{p}_{\alpha} b_{\alpha}^{\prime}=1$. This may not be an integer but we know that $b_{\alpha}^{\prime} \leq b_{\alpha}$. Thus we obtain

$$
\frac{\operatorname{ord}_{t} \partial f(\mathbf{z}(t))}{\operatorname{ord}_{t} f(\mathbf{z}(t))} \leq 1-\frac{1}{b_{\alpha}}, \quad \forall \alpha, p_{\alpha}=p_{\text {min }} .
$$


Put $B:=\max \left\{b_{j} \mid j=1, \ldots, n\right\}$ and let $I_{B}=\left\{j \mid b_{j}=B\right\}$. Thus the above estimation gives also $\theta(\mathbf{z}(t))=\frac{\operatorname{ord}_{t} \partial f(\mathbf{z}(t))}{\operatorname{ord}_{t} f(\mathbf{z}(t))} \leq 1-1 / B$.

Definition 18. The monomial $z_{j}^{b_{j}}$ is called Eojasiewicz monomial if $b_{j}=B$, i.e. $j \in I_{B}$. The monomial $z_{j}^{b_{j}}$ is called Eojasiewicz exceptional if $j \in I_{B}$ and there exists $k \neq j$ and a monomial $z_{j}^{B^{\prime}} z_{k}$ in $f(\mathbf{z})$ with $B^{\prime}<B-1$. Otherwise $z_{j}^{B}$ is called a non-exceptional Lojasiewicz monomial ([20]).

Proposition 19. If $f$ has a non-exceptional Eojasiewicz monomial, there exists an analytic curve $\mathbf{z}(t)$ so that the equality, $\theta(\mathbf{z}(t))=1-1 / B$, holds and thus

$$
\theta_{0}(f)=1-\frac{1}{B}
$$

Proof. To see this, assume that $z_{j_{0}}^{b_{j_{0}}}$ is a non-exceptional Lojasiewicz monomial. Consider the analytic curve $\mathbf{z}(t)$ which is defined by $z_{j_{0}}(t)=t$ and $z_{j}(t)=t^{N}$ for any $j \neq j_{0}$ where $N$ is a sufficiently large positive integer. Then it is easy to see that $\frac{\partial f}{\partial z_{j_{0}}}(\mathbf{z}(t))=c t^{B-1}+$ (higher terms), $c \neq 0$. If the derivative $\frac{\partial f}{\partial z_{j}}(\mathbf{z}), j \neq j_{0}$ contains a monomial $z_{j_{0}}^{a}$, it comes from the mono-

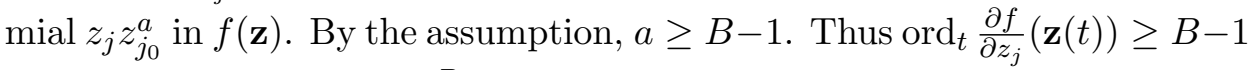
for $j \neq j_{0}$ and $f(\mathbf{z}(t))=c t^{B}, c \neq 0$. Therefore it is easy to see that the equality is satisfied.

Theorem 20. Assume that $f(\mathbf{z})$ is a convenient non-degenerate function. Then $\theta_{0}(f) \leq 1-1 / B$. Furthermore if there exists a non-exceptional Lojasiewicz monomial, the equality holds.

Example 21. Consider $f(\mathbf{z})=z_{1}^{5}+z_{1}^{3} z_{2}+z_{2}^{4}+z_{3}^{4}$. Then $B=5$ but $z_{1}^{5}$ is an exceptional Łojasiewicz monomial. In fact, Łojasiewicz exponent is given by $\theta_{0}(f)=1-\frac{1}{4}=3 / 4$.

3.2. Non-convenient case. We assume that $f(\mathbf{z})$ is non-degenerate and strongly inv-tame but we do not assume the convenience of $f$. The singularity is not necessarily isolated. Let $\mathcal{D}$ be the set of equivalent classes $[P]$ with $\operatorname{dim}[P]=n$.

For a $[P] \in \mathcal{D}$, the face function is given as a monomial function $f_{P}(\mathbf{z})=$ $c \mathbf{z}^{\nu(P)}$ and we associate the total degree $|\nu(P)|$ to $[P]$.

Main Theorem 22. Assume that $f$ is non-degenerate and strongly invtame function. Then the Eojasiewicz exponent of type (1) has the estimation:

$$
\theta_{0}(f) \leq \max \{L, \widetilde{\theta}\}
$$

where

$$
\begin{aligned}
& \widetilde{\theta}:=\max \left\{1-\hat{p}_{\text {min }}^{\prime} \mid \hat{P} \in \mathcal{W}_{+} \cup \mathcal{W}_{v}, \operatorname{dim} \Delta(P)=n-1\right\} \\
& L=\max \{1-1 /|\nu(P)| \mid[P] \in \mathcal{D}\}
\end{aligned}
$$


Proof. Consider an analytic curve $C \in \mathcal{P}$ defined by $\mathbf{z}=\mathbf{z}(t), 0 \leq t \leq 1$ and $f(\mathbf{z}(t)) \neq 0$ for $t \neq 0$. We may assume that $\mathbf{z}(t) \in \mathbb{C}^{* n}$ for $t \neq 0$ by Proposition 8. Assume that $\mathbf{z}(t)$ has the following expansion:

$$
\begin{aligned}
z_{j}(t) & =a_{j} t^{r_{j}}+(\text { higher terms }), \quad a_{j} \neq 0, j=1, \ldots, n, \\
\frac{\partial f}{\partial z_{j}}(\mathbf{z}(t)) & =\frac{\partial f_{R}}{\partial z_{j}}(\mathbf{a}) t^{d(R)-p_{j}}+(\text { higher terms }), \\
f(\mathbf{z}(t)) & =b t^{d^{\prime}}+\left(\text { higher terms) }, d^{\prime} \geq d(R) .\right.
\end{aligned}
$$

where $R=\left(r_{1}, \ldots, r_{n}\right), \mathbf{a}=\left(a_{1}, \ldots, a_{n}\right) \in \mathbb{C}^{* n}$. We use the same notation as in [18]. If $\Delta(R)$ is a vertex, $[R] \in \mathcal{D}$ and it is clear that $\frac{\partial f_{R}}{\partial z_{j}}(\mathbf{a}) \neq 0$ for any $j \in \operatorname{Var}(R)$ and $\theta(\mathbf{z}(t)) \leq 1-\frac{1}{L}$ by Lemma 10 .

Now we assume that $\operatorname{dim} \Delta(R) \geq 1$. Assume that $\frac{\partial f_{P}}{\partial z_{j}}(\mathbf{a}) \neq 0$ for some $j \in \widehat{\operatorname{Var}}(P)$, it is clear from the definition that $\theta(C(t)) \leq 1-\hat{p}_{j}$. Thus $\theta(\mathbf{z}(t)) \leq \theta(R)^{\prime}$ by the non-degeneracy and the strong inv-tameness. Thus the proof is reduced to the following assertion.

Assertion 23. Assume that $\operatorname{dim} \Delta(R) \geq 1$. Then

$$
\theta(R)^{\prime} \leq \max \left\{\theta(P)^{\prime} \mid P \succ R, \operatorname{dim} \Delta(P)=n-1, P \in \mathcal{W}_{+} \cup \mathcal{W}_{v}\right\}
$$

This is proved easily on the induction on $\operatorname{dim}[R]$, using Lemma 15 and Lemma 16. If $\operatorname{dim}[R]=1, R$ is a vertex and there are nothing to be proved. Take an admissible line segment $\operatorname{LS}(P, Q), P_{s}=(1-s) P+s Q$ and $R=P_{s_{0}}$ for some $0<s_{0}<1$. By Lemma 15 and Lemma 16, we have the estimation

$$
\theta(R)^{\prime} \leq \max \left\{\theta(P)^{\prime}, \theta(Q)^{\prime}\right\}
$$

for $P, Q \in \mathcal{W}_{+} \cup \mathcal{W}_{v}$ and the assertion holds for $R$ by the strong local invtameness. The assertion holds by the induction's hypothesis for $P$ and $Q$ if $P, Q \in \mathcal{W}_{+} \cup \mathcal{W}_{v}$. If $P$ is non-vanishing, the estimation is simply replaced by $\theta(R)^{\prime} \leq \theta(Q)^{\prime}$.

\subsection{Examples of the estimation of $\theta_{0}(f)$.}

Example 24. Consider the polynomial $f_{1}(\mathbf{z})=z_{1}^{4} z_{2}^{2}+z_{1}^{6} z_{3}+z_{2}^{6} z_{3}^{2}+z_{1}^{3} z_{3}^{6}$ considered in Example 5. We have

$$
\theta(P)^{\prime}=\frac{10}{11}, \theta(Q)^{\prime}=\frac{8}{9}, \theta(R)^{\prime}=\frac{1}{2}, \theta(S)^{\prime}=\frac{4}{5}, \theta(T)^{\prime}=\frac{5}{6}
$$

The region $A, B, C, D$ corresponds to the monomials $z_{1}^{5} z_{2}^{2}, z_{1}^{6} z_{3}, z_{2}^{6} z_{3}^{2}, z_{3}^{6} z_{1}^{3}$ respectively and these region give the bounds $6 / 7,6 / 7,7 / 8,8 / 9$ respectively. Thus $\theta_{0}\left(f_{1}\right) \leq \frac{10}{11}$ by Theorem 22 .

Remark 25. The estimation by Main Theorem 22 is not always sharp. In fact, the equality in the above estimation can not be obtained. For the weight vector $P, f_{1 P}(\mathbf{z})=z_{1}^{6} z_{3}+z_{1}^{4} z_{2}^{2}+z_{1}^{3} z_{3}^{6}$ and we see that $\frac{\partial f_{1 P}}{\partial z_{2}} \neq 0$ on $\mathbb{C}^{* 3}$. As $\hat{P}=\left(\frac{5}{33}, \frac{3}{22}, \frac{1}{11}\right)$, the real contribution for $P$ is from $\frac{\partial f_{P}}{\partial y}$. Thus 
$\theta(\mathbf{z}(t)) \leq 1-3 / 22=19 / 22$ for any $\mathbf{z}(t)$ with wt $(\mathbf{z}(t))=P$. The contribution from $Q$ is in fact sharp. Note that $f_{1 Q}$ is given by $z_{1}^{5} z_{2}^{2}+z_{1}^{6} z_{3}+z_{2}^{6} z_{3}^{2}$ and one can find $\mathbf{z}(t)$ with the coefficient vector a satisfies $\frac{\partial f_{1 Q}}{\partial z_{1}}(\mathbf{a})=\frac{\partial f_{1 Q}}{\partial z_{2}}(\mathbf{a})=0$. Thus $\theta(\mathbf{z}(t))=\frac{8}{9}$. As $\frac{8}{9}>\frac{19}{22}$, we conclude $\theta_{0}\left(f_{1}\right)=\frac{8}{9}$.

3.4. Is $\theta_{0}(f)$ a moduli invariant? For a given non-degenerate function $f(\mathbf{z})$, we ask if the Eojasiwwicz exponents are constant or not on the moduli space. For this purpose, we consider the branched poly-cyclic covering $\varphi_{2}$ : $\mathbb{C}^{n} \rightarrow \mathbb{C}^{n}$ and its lift of $f$, defined by $\varphi_{2}(\mathbf{w})=\mathbf{z}, z_{i}=w_{i}^{2}(1 \leq i \leq n)$ and put $f^{(2)}(\mathbf{w}):=\varphi^{*} f(\mathbf{w})=f\left(w_{1}^{2}, \ldots, w_{n}^{2}\right)$. More precisely we consider $f_{1}(\mathbf{z})=z_{1}^{5} z_{2}^{2}+z_{1}^{6} z_{3}+z_{2}^{6} z_{3}^{2}+z_{3}^{6} z_{1}^{3}$ in Example 1. Put $f_{1}^{(2)}(\mathbf{w}):=f_{1}\left(\varphi_{2}(\mathbf{w})\right)=$ $w_{1}^{10} w_{2}^{4}+w_{1}^{12} w_{3}^{2}+w_{2}^{12} w_{3}^{4}+w_{3}^{12} z_{1}^{6}$. The dual Newton diagram $\Gamma^{*}\left(f^{(2)}\right)$ is given by the same diagram of $\Gamma^{*}\left(f_{1}\right)$. Only change is that $d\left(K, f_{1}^{(2)}\right)=2 d\left(K, f_{1}\right)$ for any weight vector $K$. Thus in the normalized vectors, $\hat{P}, \hat{Q}, \hat{R}, \hat{S}, \hat{T}$ are to be divided by 2. By the same discussion as in the above Remark, we see that $\theta_{0}\left(f_{1}^{(2)}\right)=\frac{17}{18}$. Let $g(\mathbf{w}):=f_{1}^{(2)}(\mathbf{w})+w_{1}^{3} w_{2}^{6} w_{3}^{8}$. Note that the new monomial $w_{1}^{3} w_{2}^{6} w_{3}^{8}$ corresponds to the midpoint of the edge $C^{2)} D^{(2)}$. Here $C^{(2)}, D^{(2)}$ are the lift of $C, D$ in $\Gamma\left(f^{(2)}\right)$. Thus the dual Newton diagram of $g$ is the same with $\Gamma^{*}\left(f^{(2)}\right)$. By the result of [6], the family $g_{t}(\mathbf{w}):=$ $f_{1}^{(2)}(\mathbf{w})+t w_{1}^{3} w_{2}^{6} w_{3}^{8}$ is non-degenerate and strongly locally inv-tame except a finite exceptional $t$ 's. The exceptional set $S$ is the union of $\{ \pm 2\}$ from the non-degeneracy of $g_{t, T}$ and possibly some more $t$ 's from the non-degeneracy of $g_{t \hat{P}}$. Actually $f_{t \hat{P}}=0$ is non-singular in $\mathbb{C}^{* 3}$ for $t \neq \pm 2$ as we can see by a direct calculation, $\frac{\partial g_{t \hat{P}}}{\partial z_{1}}=\frac{\partial g_{t \hat{P}}}{\partial z_{2}}=\frac{\partial g_{t \hat{P}}}{\partial z_{3}}=0$ has no solution in $\mathbb{C}^{* 3}$. Thus $S=\{ \pm 2\}$. This family has a canonical Whitney regular stratification and $V\left(f^{(2)}\right)$ and $V(g)$ are topologically equivalent for any $t \in \mathbb{C} \backslash S$. We assert $\theta_{0}(g)=\frac{21}{22}\left(=1-\frac{6}{132}\right)$ which comes from the vertex $P=(10,9,6)$ with $d(P, g)=132$. Thus $\theta_{0}(g)>\theta_{0}\left(f_{1}^{(2)}\right)$ and $\theta_{0}$ is not constant on the moduli space of $g$. Here we mean by moduli the space of functions with fixed Newton boundary and local tameness. For example, we can choose the following curve which satisfies $\frac{\partial g_{P}}{\partial w_{1}}=\frac{\partial g_{P}}{\partial w_{2}}=0$ :

$$
w_{1}(s)=\frac{1}{2} \sqrt[6]{3} \sqrt{2} \sqrt[6]{-1} s^{10}, w_{2}(s)=\sqrt[6]{-\frac{1}{8} i \sqrt{6}} s^{9}, w_{3}(s)=s^{6} .
$$

We can see that

$$
\begin{aligned}
& \operatorname{ord}_{s} \partial g(\mathbf{w}(s))=126, \quad \operatorname{ord}_{s} g(\mathbf{w}(s))=132 \\
& \theta(\mathbf{w}(s))=\frac{126}{132}=\frac{21}{22} .
\end{aligned}
$$

Unfortunately $g_{t}$ has 1-dimensional singularity.

For isolated singularity case, this does not happen. In fact, Brzostowski proved the Eojasiewicz exponent $\eta_{0}(f)$ of the Łojasiewicz inequality of type (2) is constant on the moduli space of functions with fixed Newton boundary 
and an isolated singularity at the origin (Theorem 1, 4]). On the other hand, $\theta_{0}(f)$ and $\eta_{0}(f)$ are related by $\theta_{0}(f)=\eta_{0}(f) /\left(1+\eta_{0}(f)\right)$ by Teissier [23]. I thank Professor Tadeusz Krasiński for this information.

Example 26. Consider the simplicial weighted homogeneous polynomial $f_{2}(\mathbf{z})=z_{1}^{a} z_{2}^{2}+z_{2}^{b} z_{3}^{2}+z_{3}^{c} z_{1}^{2}$ in Example 2. Then normalized weight is given as $\hat{P}=\left(\frac{4-2 c+b c}{a b c+8}, \frac{a c+4-2 a}{a b c+8}, \frac{a b+4-2 b}{a b c+8}\right)$. Suppose $c \geq a, b$. Then the contribution from $\hat{P}$ is $1-\frac{a b-2 b+4}{a b c+8}$. The contribution from $\hat{R}, \hat{S}, \hat{T}$, which are $\left.\theta(\hat{R})^{\prime}, \theta(\hat{S})^{\prime}, \theta(\hat{T})^{\prime}\right)$, are given by $1-1 / c, 1-1 / b, 1-1 / a$ respectively by Theorem 22 but these estimation is not sharp. For example, $f_{\hat{R}}=z_{1}^{a} z_{2}^{2}+z_{3}^{c} z_{1}^{2}$ and $\frac{\partial f_{\hat{R}}}{\partial z_{2}}$ can not be zero on $\mathbb{C}^{* 3}$. Thus the real contribution is $1-1 / 2=1 / 2$. The same is true for $\hat{S}, \hat{T}$. Thus $\theta_{0}(f)=1-\frac{a b-2 b+4}{a b c+8}$.

Example 27. Consider $f_{3}(\mathbf{z})=z_{1}^{4} z_{2}^{2}+z_{2}^{4} z_{3}^{2}+z_{3}^{4} z_{1}^{2}+z_{1} z_{2} z_{3}$ of Example 3 (See Figure 4). This polynomial has no compact face of dimension 2 in $\Gamma(f)$. We observe that $\theta\left(S_{1}\right)^{\prime}, \theta\left(T_{1}\right)^{\prime}, \theta\left(R_{1}\right)^{\prime}=\frac{1}{2}$ and $\theta\left(S_{2}\right)^{\prime}, \theta\left(T_{2}\right)^{\prime}, \theta\left(R_{2}\right)^{\prime}=$ $\frac{3}{4}$. $\mathcal{D}$ contains 4 regions. The pentagon with vertices $S_{2}, S_{1}, T_{2}, T_{1}, R_{2}, R_{1}$ contribute by $\frac{2}{3}$. The other triangles contribute by $\frac{5}{6}$. Thus we have $\theta_{0}\left(f_{3}\right) \leq$ $\frac{5}{6}$. In fact, $\theta_{0}\left(f_{3}\right)=\frac{5}{6}$. To see this, consider the triangle region $S_{1} T_{2} E_{3}$ in Figure 4 and take an analytic curve, for example, $\mathbf{z}(t)=\left(t, t, t^{N}\right)$ for a sufficiently large. Then the weight vector is given by $P=(1,1, N)$ or $\hat{P}=\left(\frac{1}{6}, \frac{1}{6}, \frac{N}{6}\right)$ and $f_{3 P}=z_{1}^{4} z_{2}^{2}$ and $\theta(\mathbf{z}(t))=\frac{5}{6}$.

3.5. $\theta_{0}(f)$ does not behave like Milnor numbers. We give another example of a delicate behavior of $\theta_{0}(f)$. Assume that $f(\mathbf{z}), g(\mathbf{z})$ have isolated singularities at the origin and they are non-degenerate. Let $\Gamma_{-}(f), \Gamma_{-}(g)$ be the cones of $\Gamma(f), \Gamma(g)$ with the origin. Assume that $\Gamma_{-}(g) \supsetneq \Gamma_{-}(f)$ and $\Gamma(f) \cap \mathbb{R}^{I}=\Gamma(g) \cap \mathbb{R}^{I}$ for any $I \subsetneq\{1, \ldots, n\}$. Then by Kouchnirenko's theorem $([10])$, the Milnor numbers satisfies the inequality: $\mu(g)>\mu(f)$. This is not always true for $\theta_{0}(g)$ and $\theta_{0}(f)$.

As an example, consider $g_{4}(\mathbf{z})=z_{1}^{9} z_{2}+z_{2}^{10} z_{3}+z_{3}^{11} z_{1}$ and $f_{4}(\mathbf{z})=g_{4}(\mathbf{z})+$ $z_{1}^{2} z_{2}^{2} z_{3}^{2}$. Note that $\Gamma_{-}\left(g_{4}\right) \supset \neq \Gamma_{-}\left(f_{4}\right)$. First, their Milnor numbers are given as $\mu\left(g_{4}\right)=990$ and $\mu\left(f_{4}\right)=543$. Their dual Newton boundaries are given as Figure 5 and Figure 6. Łojasiewicz exponent is given as $\theta_{0}\left(g_{4}\right)=\frac{910}{991}=$ $0.91 \cdots$. On the other hand, $\theta_{0}\left(f_{4}\right) \leq \frac{95}{101}=0.94 \cdots$ which comes from $T_{3}=\left(\frac{35}{101}, \frac{19}{202}, \frac{6}{101}\right)$. In fact, we can show that the equality $\theta_{0}(f)=\frac{95}{101}$ is taken by the following curve:

$$
z_{1}(t)=b_{1} t^{70}, z_{2}(t)=b_{2} t^{19}, z_{3}(t)=b_{3} t^{12}
$$

where $\mathbf{b} \in \mathbb{C}^{3 *}$ satisfies the equality

$$
\begin{aligned}
& \frac{\partial f_{T_{3}}}{\partial z_{1}}(\mathbf{b})=\frac{\partial f_{T_{3}}}{\partial z_{2}}(\mathbf{b})=0 \\
& f_{T_{3}}(\mathbf{z})=z_{2}^{10} z_{3}+z_{3}^{11} z_{1}+z_{1}^{2} z_{2}^{2} z_{3}^{2} .
\end{aligned}
$$


Then we see that $\operatorname{ord}_{t} \partial f_{4}(\mathbf{z}(t))=190, \operatorname{ord}_{t} f_{4}(\mathbf{z}(t))=202$. For example, we can take

$$
b_{1}=\sqrt[6]{\frac{5}{16}}, b 2:=\sqrt[12]{\frac{1}{20}}, b_{3}=-1 .
$$

Thus we have the inequality: $\theta_{0}\left(f_{4}\right)>\theta_{0}\left(g_{4}\right)$, while $\mu\left(f_{4}\right)<\mu\left(g_{4}\right)$.

$$
\begin{aligned}
& \hat{P}=\left(\frac{100}{991}, \frac{91}{991}, \frac{81}{991}\right) \\
& \hat{S}_{1}=\left(0,1 \frac{1}{11}\right), \hat{S}_{2}=\left(\frac{1}{9}, 0,1\right) \\
& \hat{S}_{3}=\left(1, \frac{1}{10}, 0\right) \\
& g_{4}=z_{1}^{9} z_{2}+z_{2}^{10} z_{3}+z_{3}^{11} z_{1}
\end{aligned}
$$

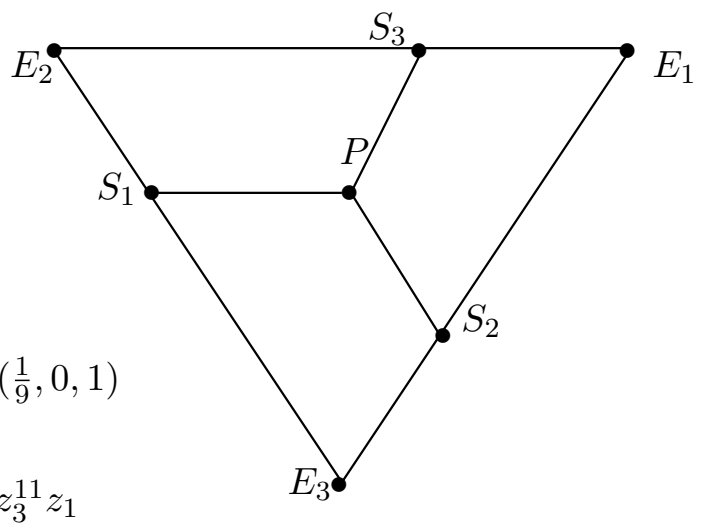

Figure 5. $\Gamma^{*}\left(g_{4}\right)$ 


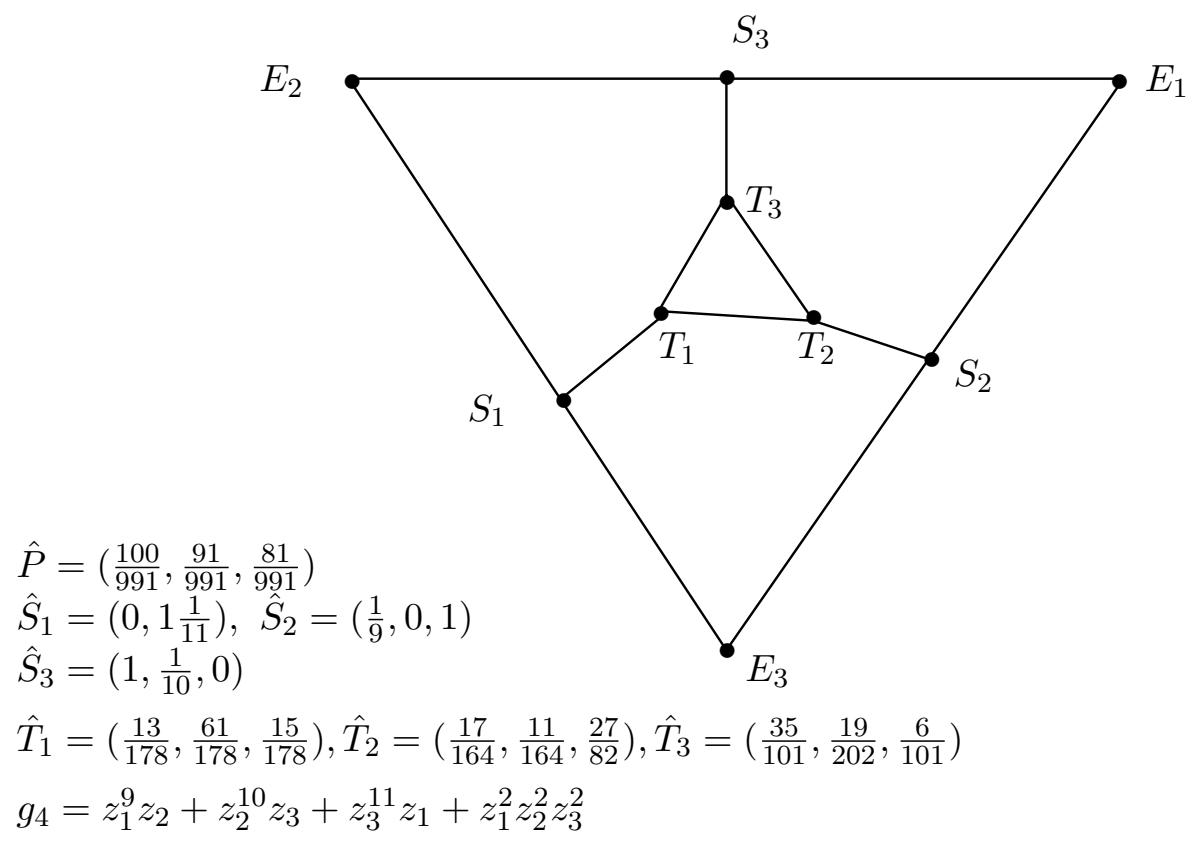

FiguRe $6 . f_{4}(\mathbf{z})$

\section{4. ŁOJASIEWICZ EXPONENTS OF NON-IRREDUCIBLE FUNCTIONS}

In this section, we study Łojasiewicz exponents of reducible functions which are associated with non-degenerate complete intersection varieties.

\subsection{A function associated with a convenient non-degenerate com-} plete intersection variety. We consider a family of functions $\mathcal{F}:=\left\{f_{1}, \ldots, f_{k}\right\}$. We say $\mathcal{F}$ is a defining family of convenient non-degenerate complete intersection varieties if each $f_{\alpha}$ is a convenient non-degenerate function such that for each $I \subset\{1, \ldots, k\}, V(I):=\left\{\mathbf{z} \in \mathbb{C}^{n} \mid f_{i}(\mathbf{z})=0, i \in I\right\}$ is a non-degenerate complete intersection variety in the sense of Khovanskii $([9,[1])$. Namely for any strictly positive weight vector $P$, the variety $V_{I}(P)^{*}:=\left\{\mathbf{z} \in \mathbb{C}^{* n}\left|f_{j P}(\mathbf{z})=0\right| j \in I\right\}$ is a smooth complete intersection variety in $\mathbb{C}^{* n}$. We consider the product function $f=f_{1} \ldots f_{k}$ and we call $f$ as the function associated with the family $\mathcal{F}$. Note that $f$ is also a convenient function. In [7], we considered a similar family, which we called $a$ Newton-admissible family $\mathcal{F}=\left\{f_{1 s}, \ldots, f_{k s}\right\}$ with one parameter $s \in \mathbb{C}$ and the associated product function $f_{s}=f_{1 s} \ldots f_{k s}$ in $s \in \mathbb{C}$ to study the topological stability of the one-parameter family of hypersurfaces $V_{s}=f_{s}^{-1}(0)$.

In this paper, we assume that each function $f_{j}$ is convenient and has no parameter $s$. However the argument below can be done in the exact same way for one parameter family if their Newton boundary $\Gamma\left(f_{j s}\right)$ is independent of $s$. As in $\S 3.1$, we put $b_{\alpha, j}, 1 \leq \alpha \leq k, 1 \leq j \leq n$ be the unique point of 
$\Gamma\left(f_{\alpha}\right)$ on $z_{j}$-axis. So $z_{j}^{b_{\alpha}, j}$ is a monomial of $f_{\alpha}(\mathbf{z})$ with non-zero coefficient. We will give an explicit estimation for $\theta_{0}(f)$. The hypersurface $V(f)$ has $k$ irreducible components $V\left(f_{\alpha}\right), \alpha=1, \ldots, k$ and $V(f)$ has non-isolated singularities if $k \geq 2$. The singular locus of $V(f)$ is the union $\cup_{i \neq j} V\left(f_{i}, f_{j}\right)$. Here $V\left(f_{i}, f_{j}\right):=\left\{f_{i}=f_{j}=0\right\}$.

We are interested in the estimation of the Łojasiewicz component of $f$. As in the previous section, we start to consider an analytic curve $\mathbf{z}(t), 0 \leq t \leq 1$ which starts from the origin and $\mathbf{z}(t) \in \mathbb{C}^{* n} \backslash V(f)$ for $t \neq 0$ and we consider its Taylor expansion:

$$
z_{j}(t)=a_{j} t^{p_{j}}+(\text { higher terms }), \quad p_{j}>0, a_{j} \neq 0, j=1, \ldots, n .
$$

(Actually we also consider the case $\mathbf{z}(t) \in \mathbb{C}^{* J}(t \neq 0), J \subset\{1, \ldots, k\}$ such that $f^{J} \mathrm{~s}$ not constantly zero. However the estimation is reduced to the case $\mathbb{C}^{* n}$ by Proposition 8.) Put $P=\left(p_{1}, \ldots, p_{n}\right)$ and $\mathbf{a}=\left(a_{1}, \ldots, a_{n}\right)$ as before. Let $z_{j}^{b_{\alpha j}}$ be the monomial of $\Gamma\left(f_{\alpha}\right)$ on the $j$-th axis for $j=1, \ldots, n$ and $1 \leq \alpha \leq k$. Let

$$
\begin{aligned}
f_{\alpha}(\mathbf{z}(t)) & =c_{\alpha} t^{d_{\alpha}}+(\text { higher terms }), c_{\alpha} \neq 0,1 \leq \alpha \leq k, \\
f(\mathbf{z}(t)) & =c t^{d}+(\text { higher terms }), c \neq 0 \\
d_{\alpha} & \geq d\left(P, f_{\alpha}\right), \quad d \geq d(P, f)=\sum_{\alpha} d\left(P, f_{\alpha}\right) .
\end{aligned}
$$

We use this expansion assumption throughout this paper. Note that $d=$ $\sum_{\alpha=1}^{k} d_{\alpha}$ and $c=c_{1} \cdots c_{k}$. Put $e_{\alpha}:=d\left(P, f_{\alpha}\right)$ and $e=\sum_{\alpha=1}^{k} e_{\alpha}$. We observe that

$$
\begin{aligned}
\frac{\partial f_{\alpha}}{\partial z_{j}}(\mathbf{z}(t)) & =\frac{\partial f_{\alpha P}}{\partial z_{j}}(\mathbf{a}) t^{e_{\alpha}-p_{j}}+(\text { higher terms }) \\
\partial f(\mathbf{z}(t)) & =\sum_{\alpha=1}^{k}\left(\prod_{\beta \neq \alpha} f_{\beta}(\mathbf{z}(t))\right) \partial f_{\alpha}(\mathbf{z}(t)),
\end{aligned}
$$

Therefore by (12) and (13),

$$
\begin{aligned}
\frac{\partial f}{\partial z_{j}}(\mathbf{z}(t)) & =\sum_{\alpha=1}^{k}\left(\prod_{\beta \neq \alpha} f_{\beta}(\mathbf{z}(t))\right) \frac{\partial f_{\alpha}}{\partial z_{j}}(\mathbf{z}(t)) \\
& =\sum_{\alpha=1}^{k}\left\{\frac{\partial f_{\alpha P}}{\partial z_{j}}(\mathbf{a}) \frac{c}{c_{\alpha}} t^{d-d_{\alpha}+e_{\alpha}-p_{j}}+\text { (higher terms) }\right\}
\end{aligned}
$$

Put $B_{j}:=\sum_{\alpha=1}^{k} b_{\alpha, j}$ and $B=\max \left\{B_{j} \mid j=1, \ldots, n\right\}$. Note that $z_{j}^{B_{j}}$ has a non-zero coefficient in the expansion of $f(\mathbf{z})$ and it corresponds to the unique point of $\Gamma(f)$ on the $z_{j}$-axis. Let $p_{\min }:=\min \left\{p_{j} \mid j=1, \ldots, n\right\}$, $I_{\text {min }}=\left\{j \mid p_{j}=p_{\min }\right\}$. 
4.1.1. Case 1. a is generic. We consider the case $\mathbf{a}$ is generic so that $f_{P}(\mathbf{a})=$ $\prod_{\alpha=1}^{k} f_{\alpha P}(\mathbf{a}) \neq 0$. This implies that $d_{\alpha}=e_{\alpha}$ for any $\alpha=1, \ldots, k$. As $f_{P}(\mathbf{z})$ is a weighted homogeneous polynomial of degree $e=\sum_{\alpha} e_{\alpha}$ with respect to the weight vector $P, 0$ is the only possible critical value of $f_{P}$. Thus $d=e=\sum_{\alpha=1}^{k} e_{\alpha}$ and $\partial f_{P}(\mathbf{a}) \neq \mathbf{0}$ and

$$
\frac{\operatorname{ord}_{t} \partial f(\mathbf{z}(t))}{\operatorname{ord}_{t} f(\mathbf{z}(t))} \leq \frac{e-p_{\text {min }}}{e}=1-\frac{p_{\text {min }}}{e}
$$

We define a rational number $b_{\alpha, j}^{\prime}$ by $b_{\alpha, j}^{\prime} p_{j}=e_{\alpha}$ for $j \in I_{\text {min }}$. Then $p_{j} B_{j}^{\prime}=e$ where $B_{j}^{\prime}=\sum_{\alpha=1}^{k} b_{\alpha, j}^{\prime}$ and $j_{0}$ is fixed in $I_{\min }$. Thus $b_{\alpha, j_{0}}^{\prime} \leq b_{\alpha, j_{0}}$. Note the equality $p_{j_{0}} \sum_{\alpha=1}^{k} b_{\alpha, j_{0}}^{\prime}=p_{j_{0}} B_{j_{0}}^{\prime}=e$. Thus the above estimation implies

$$
\frac{\operatorname{ord}_{t} \partial f(\mathbf{z}(t))}{\operatorname{ord}_{t} f(\mathbf{z}(t))} \leq 1-\frac{1}{B_{j_{0}}^{\prime}} \leq 1-\frac{1}{B_{j_{0}}} \leq 1-\frac{1}{B}
$$

4.1.2. Case 2. a is not generic. This case is more complicated. We consider non-generic coefficient vector $\mathbf{a}$. So we assume that there exists $\alpha, 1 \leq \alpha \leq k$ such that $f_{\alpha P}(\mathbf{a})=0$. Consider the defect numbers $d_{j}^{\prime}:=d_{j}-e_{j}, 1 \leq j \leq k$ and changing the numbering of $f_{j}, 1 \leq j \leq k$ if necessary, we assume for simplicity

$$
d_{1}^{\prime} \leq d_{2}^{\prime} \leq \cdots \leq d_{\ell-1}^{\prime}<d_{\ell}^{\prime}=\cdots=d_{k}^{\prime}
$$

for some $\ell, 1 \leq \ell \leq k$. Therefore $\ell:=\min \left\{\alpha \mid d_{\alpha}=d_{k}^{\prime}\right\}$. Note that $f_{\alpha P}(\mathbf{a}) \neq$ 0 if and only if $d_{\alpha}^{\prime}=0$. In particular $f_{\alpha P}(\mathbf{a})=0$ as $d_{\alpha}^{\prime}>0$ for $\ell \leq \alpha \leq k$. We have the estimation:

$$
\begin{aligned}
\operatorname{ord}_{t} & \left\{\left(\prod_{\beta \neq \alpha} f_{\beta}(\mathbf{z}(t))\right) \frac{\partial f_{\alpha}}{\partial z_{j}}(\mathbf{z}(t))\right\} \\
\geq & d-d_{\alpha}+\left(e_{\alpha}-p_{j}\right)=d-d_{\alpha}^{\prime}-p_{j}, 1 \leq j \leq n .
\end{aligned}
$$

Note that

$$
\begin{aligned}
d-d_{1}^{\prime}-p_{j} & \geq \cdots \geq d-d_{\ell-1}^{\prime}-p_{j} \\
& >d-d_{\ell}^{\prime}-p_{j}=\cdots=d-d_{k}^{\prime}-p_{j} .
\end{aligned}
$$

and finally we have the expression:

$$
\frac{\partial f}{\partial z_{j}}(\mathbf{z}(t))=\left(\sum_{\alpha=\ell}^{k} \frac{c}{c_{\alpha}} \frac{\partial f_{\alpha P}}{\partial z_{j}}(\mathbf{a})\right) t^{d-d_{\ell}^{\prime}-p_{j}}+\text { (higher terms). }
$$

Assertion 28. There exists some $j_{0}$ such that $\operatorname{ord}_{t} \frac{\partial f}{\partial z_{j_{0}}}(\mathbf{z}(t))=d-d_{\ell}^{\prime}-p_{j_{0}}$.

Proof. By the assumption, we have $d_{\ell}^{\prime}=\cdots=d_{k}^{\prime}$. For the proof of the assertion, we use the non-degeneracy assumption of the complete intersection variety $V_{I}(P)^{*}:=\left\{\mathbf{z} \in \mathbb{C}^{* n} \mid f_{\alpha P}(\mathbf{z})=0, \alpha \in I\right\}$ with $I=\{\ell, \ldots, k\}$. By the assumption, $\mathbf{a} \in V_{I}(P)^{*}$. Assume that $\sum_{\alpha=\ell}^{k} \frac{c}{c_{\alpha}} \frac{\partial f_{\alpha P}}{\partial z_{j}}(\mathbf{a})=0$ for any $j$. This implies $\sum_{\alpha=\ell}^{k} \frac{c}{c_{\alpha}} \partial f_{\alpha P}(\mathbf{a})=0$ and it gives a non-trivial linear relation 
among gradient vectors $\partial f_{\ell P}(\mathbf{a}), \ldots, \partial f_{k P}(\mathbf{a})$ which is a contradiction to the non-degeneracy of the complete intersection assumption $V_{I}(P)^{*}$.

Thus there exists $j_{0}, 1 \leq j_{0} \leq n$ so that

$$
\sum_{\alpha=\ell}^{k} \frac{c}{c_{\alpha}} \frac{\partial f_{\alpha P}}{\partial z_{j_{0}}}(\mathbf{a}) \neq 0, \text { that is, } \operatorname{ord}_{t} \frac{\partial f}{\partial z_{j_{0}}}(\mathbf{z}(t))=d-d_{\ell}^{\prime}-p_{j_{0}} .
$$

Consider the integers:

$$
D_{\alpha-1}^{\prime}:=d_{1}^{\prime}+\cdots+d_{\alpha-1}^{\prime}, 1 \leq \alpha \leq k .
$$

We have

$$
\begin{aligned}
\operatorname{ord}_{t} f(\mathbf{z}(t)) & =d_{1}+\cdots+d_{k}=D_{\ell-1}^{\prime}+(k-\ell) d_{\ell}^{\prime}+e, \\
\operatorname{ord}_{t} \partial f(\mathbf{z}(t)) & =\inf \left\{\operatorname{ord}_{t} \frac{\partial f}{\partial z_{j}}(\mathbf{z}(t)) \mid 1 \leq j \leq n\right\} \\
& \leq \sup \left\{d-d_{\ell}^{\prime}-p_{j} \mid 1 \leq j \leq n\right\} \\
& \leq D_{\ell-1}^{\prime}+(k-\ell-1) d_{\ell}^{\prime}+e-\frac{e}{B}
\end{aligned}
$$

Here we have used the equality $p_{j} \geq e / B_{j} \geq e / B$ and $d_{\alpha}=d_{\alpha}^{\prime}+e_{\alpha}$, in particular $d_{\alpha}=d_{\ell}^{\prime}+e_{\alpha}$ for $\alpha \geq \ell$. Thus we have

$$
\theta(\mathbf{z}(t))=\frac{\operatorname{ord}_{t} \partial f(z(t))}{\operatorname{ord}_{t} f(\mathbf{z}(t))} \leq F_{\ell}
$$

where $F_{\ell}$ is defined by the following:

$$
F_{\ell}:=\frac{D_{\ell-1}^{\prime}+(k-\ell-1) d_{\ell}^{\prime}+e-e / B}{D_{\ell-1}^{\prime}+(k-\ell) d_{\ell}^{\prime}+e} .
$$

Note that under the assumption that $\mathbf{z}(t)$ has the weight vector $P$ and (17), $d_{1}^{\prime}, \ldots, d_{\ell}^{\prime}$ are not constant but the other numbers are constant. Originally $d_{\ell}^{\prime}$ is an integer but we extend to real numbers so that $F_{\ell}$ is a function of $d_{\ell}^{\prime}$ on the interval $\left[d_{\ell-1}^{\prime}, \infty\right)$, fixing $d_{1}^{\prime}, \ldots, d_{\ell-1}^{\prime}$. Note that $F_{0}=1-1 / B$.

4.2. Comparison with $F_{\ell-1}$. We want to show $F_{\ell} \leq 1-1 / B$. We assert that $F_{\ell}$ is monotone decreasing function of $d_{\ell}^{\prime}$, fixing $d_{1}^{\prime}, \ldots, d_{\ell-1}^{\prime}$ where $d_{j}^{\prime}:=d_{j}-e_{j}$. Here $d_{\ell}^{\prime}$ moves on the interval $\left[d_{\ell-1}^{\prime}, \infty\right)$. In fact, the differential of the right hand side in $d_{\ell}^{\prime}$ is given as

$$
\frac{\partial F_{\ell}}{\partial d_{\ell}^{\prime}}=\frac{-D_{\ell-1}^{\prime}-e+(k-\ell) e / B}{\left(D_{\ell-1}^{\prime}+(k-\ell) d_{\ell}^{\prime}+e\right)^{2}}
$$

We assert that

Lemma 29. $F_{\ell}$ is monotone decreasing function of $d_{\ell}^{\prime}$ as

$$
\frac{\partial F_{\ell}}{\partial d_{\ell}^{\prime}} \leq 0
$$


Proof. The numerator of the differential $\partial F_{\ell} / \partial d_{\ell}^{\prime}$ can be estimated as

$$
\begin{aligned}
-D_{\ell-1}^{\prime}-e+(k-\ell) e / B & =-D_{\ell-1}^{\prime}-e\left(1-\frac{k-\ell}{B}\right) \\
& \leq 0 .
\end{aligned}
$$

Here we have used the obvious inequality $B \geq k$.

Thus putting $d_{\ell}^{\prime}=d_{\ell-1}^{\prime}$, we get the estimation $\theta_{0}(f) \leq F_{\ell} \leq F_{\ell-1}$ where $F_{\ell-1}$ is obtained by substituting $d_{\ell}^{\prime}=d_{\ell-1}^{\prime}$ :

$$
F_{\ell-1}:=\left.F_{\ell}\right|_{d_{\ell}^{\prime}=d_{\ell-1}^{\prime}}=\frac{D_{\ell-2}^{\prime}+(k-\ell) d_{\ell-1}^{\prime}+e-e / B}{D_{\ell-2}^{\prime}+(k-\ell+1) d_{\ell-1}^{\prime}+e}
$$

where $D_{\ell-2}^{\prime}=d_{1}^{\prime}+\cdots+d_{\ell-2}^{\prime}, e=e_{1}+\cdots+e_{k}$.

4.3. Łojasiewicz exponents for the product functions. Now we are ready to state our main result for the product function.

Main Theorem 30. Let $f=f_{1} \cdots f_{k}$ be the product function associated to a generating family of convenient non-degenerate complete intersection varieties $\mathcal{F}=\left\{f_{1}, \ldots, f_{k}\right\}$. Then the Eojasiewicz exponent of type (1) satisfies the inequality: $\theta_{0}(f) \leq 1-1 / B$ and the equality holds if $f$ has a Eojasiewicz non-exceptional monomial.

Proof. Continuing the above argument repeatedly, $\theta_{0}(f)$ can be estimated by assuming $d_{\ell}^{\prime}=\cdots=d_{1}^{\prime}$ as follows:

$$
F_{\ell} \leq F_{\ell-1} \leq \cdots \leq F_{1}=\frac{(k-1) d_{1}^{\prime}+e-e / B}{(k-1) d_{1}^{\prime}+e} .
$$

As $F_{1}$ is also a monotone increasing function of $d_{1}^{\prime}$, putting $d_{1}^{\prime}=0$, we conclude $F_{\ell} \leq F_{0}=1-1 / B$. This implies

$$
\theta_{0}(f) \leq \frac{e-e / B}{e}=1-\frac{1}{B}
$$

For the existence of the curve attaining the equality under the assumption of the existence of Łojasiewicz non-exceptional monomial, we do the same argument as in $\S 3.1$.

4.4. Generalization to non-reduced functions. We will show that Łojasiewicz exponent of a non-reduced expression is determined by the reduced one. First suppose that $f$ is a reduced function and let $g(\mathbf{z})=f^{m}(\mathbf{z})$. Let $\mathbf{z}(t), 0 \leq t \leq 1$ be an analytic curve starting from the origin and $\mathbf{z}(t) \in$ $\mathbb{C}^{n} \backslash V(f)$ for $t \neq 0$ as before. Then we observe that

$$
\operatorname{ord}_{t} \frac{\partial g}{\partial z_{j}}(\mathbf{z}(t))=(m-1) \operatorname{ord}_{t} f(\mathbf{z}(t))+\operatorname{ord}_{t} \frac{\partial f}{\partial z_{j}}(\mathbf{z}(t)) .
$$

To distinguish two Lojasiewicz exponents of $f$ and $g$, we put

$$
\theta_{f}(\mathbf{z}(t)):=\frac{\operatorname{ord}_{t} \partial f(\mathbf{z}(t))}{\operatorname{ord}_{t} f(\mathbf{z}(t))}, \theta_{g}(\mathbf{z}(t)):=\frac{\operatorname{ord}_{t} \partial g(\mathbf{z}(t))}{\operatorname{ord}_{t} g(\mathbf{z}(t))} .
$$


In particular, we have the equality

$$
\begin{aligned}
\theta_{g}(\mathbf{z}(t)) & =\frac{\operatorname{ord}_{t} \frac{\partial g}{\partial z_{j}}(\mathbf{z}(t))}{\operatorname{ord}_{t} g(\mathbf{z}(t))} \\
& =\frac{(m-1) \operatorname{ord}_{t} f(\mathbf{z}(t))+\operatorname{ord}_{t} \frac{\partial f}{\partial z_{j}}(\mathbf{z}(t))}{m \operatorname{ord}_{t} f(\mathbf{z}(t))} \\
& =\frac{m-1}{m}+\frac{1}{m} \theta_{f}(\mathbf{z}(t))
\end{aligned}
$$

Thus we have

Proposition 31. The Eojasiewicz exponents of $f$ and $g=f^{m}$ are related by the equality:

$$
\theta_{0}(g)=\frac{m-1}{m}+\frac{1}{m} \theta_{0}(f) .
$$

This observation can be generalized to our product function $f$. discussed in $\S 4.1$. We consider a defining family $\mathcal{F}=\left\{f_{1}, \ldots, f_{k}\right\}$ of convenient non-degenerate complete intersection varieties as $\S 4.1$. Let $f(\mathbf{z}):=$ $f_{1}(\mathbf{z}) \cdots f_{k}(\mathbf{z})$. We consider also non-reduced product function

$$
g(\mathbf{z})=f_{1}^{m_{1}}(\mathbf{z}) \cdots f_{k}^{m_{k}}(\mathbf{z})
$$

where $m_{1}, \ldots, m_{k}$ are positive integers. Let $\mathbf{z}(t)$ be an analytic curve expanded as (11), (12) and (13). We use the same notations of numbers $e_{j}, d_{j}, d_{j}^{\prime}, e$. We define new integers $\widetilde{d}_{j}, \widetilde{e}_{j}, \widetilde{d}, \widetilde{e}$ and complex numbers $\widetilde{c}_{\alpha}, \widetilde{c}, \widetilde{d}$ as

$$
\begin{aligned}
& \widetilde{d}_{\alpha}=m_{\alpha} d_{\alpha}, \widetilde{e}_{\alpha}=m_{\alpha} e_{\alpha}, \widetilde{d}=\sum_{\alpha=1}^{k} m_{\alpha} d_{\alpha}, \widetilde{e}=\sum_{\alpha=1}^{k} m_{\alpha} e_{\alpha} \\
& \widetilde{c}_{\alpha}=c_{\alpha}^{m_{\alpha}}, \widetilde{c}=\prod_{\alpha=1}^{k} c_{\alpha}^{m_{\alpha}}
\end{aligned}
$$

so that

$$
\begin{aligned}
g_{\alpha}(\mathbf{z}(t)) & =f_{\alpha}(\mathbf{z}(t))^{m_{\alpha}}=\widetilde{c}_{\alpha} t^{\widetilde{d}_{\alpha}}+(\text { higher terms }), 1 \leq \alpha \leq k \\
g(\mathbf{z}(t)) & =\widetilde{c} t^{\widetilde{d}}+(\text { higher terms }) .
\end{aligned}
$$

We work under the same assumption (17):

$$
d_{1}^{\prime} \leq d_{2}^{\prime} \cdots \leq d_{\ell-1}^{\prime}<d_{\ell}^{\prime}=\cdots=d_{k}^{\prime} .
$$

We proceed by the exact same argument as the one in the reduced case. The equality (19) is replaced as

$$
\begin{aligned}
\frac{\partial g}{\partial z_{j}}(\mathbf{z}(t)) & =\sum_{\alpha=1}^{k}\left(\left(\frac{m_{\alpha}}{f_{\alpha}} g\right)(\mathbf{z}(t)) \frac{\partial f_{\alpha}}{\partial z_{j}}(\mathbf{z}(t))\right) \\
& =\left(\sum_{\alpha=\ell}^{k} \frac{m_{\alpha} \widetilde{c}}{\widetilde{c}_{\alpha}} \frac{\partial f_{\alpha P}}{\partial z_{j}}(\mathbf{a})\right) t^{\widetilde{d}-d_{\ell}^{\prime}-p_{j}}+\text { (higher terms) }
\end{aligned}
$$


Define $\widetilde{D}_{\alpha}$ in the same manner as in $\S 4.1$ :

$$
\widetilde{D}_{\alpha}=\widetilde{d}_{1}+\cdots+\widetilde{d}_{\alpha}=\sum_{i=1}^{\alpha} m_{i} d_{i} .
$$

$\widetilde{B}_{j}$ corresponds the point $\Gamma(g) \cap\left\{z_{j}\right.$-axis $\}$ which is equal to $\sum_{\alpha=1}^{k} m_{\alpha} b_{\alpha, j}$ and $\widetilde{B}$ is the maximum of $\left\{\widetilde{B}_{1}, \ldots, \widetilde{B}_{n}\right\}$. So $p_{\min } \widetilde{B} \geq \widetilde{e}$. As the gradient vectors

$$
\left\{\frac{\partial f_{\alpha P}}{\partial z_{j}}(\mathbf{a}) \mid \alpha=\ell, \ldots, k\right\}
$$

are linearly independent by the non-degeneracy of the intersection variety $V_{I}^{*}(P)$ (see the proof of Assertion 28), we have

$$
\begin{aligned}
& \operatorname{ord} g(\mathbf{z}(\mathrm{t}))=\widetilde{d}_{1}+\cdots+\widetilde{d}_{k}=\widetilde{D}_{\ell-1}^{\prime}+\left(m_{\ell}+\cdots+m_{k}\right) d_{\ell}^{\prime}+\widetilde{e}, \\
& \operatorname{ord} \partial \mathrm{g}(\mathbf{z}(\mathrm{t})) \leq \widetilde{d}-d_{\ell}^{\prime}-p_{\min } \leq \widetilde{D}_{\ell-1}^{\prime}+\left(m_{\ell}+\cdots+m_{k}-1\right) d_{\ell}^{\prime}+\widetilde{e}-\frac{\widetilde{e}}{\widetilde{B}} .
\end{aligned}
$$

Thus we can modify equality (20) as :

$$
\theta_{g}(\mathbf{z}(t))=\frac{\operatorname{ord}_{t} \partial g(\mathbf{z}(t))}{\operatorname{ord}_{t} g(\mathbf{z}(t))} \leq \widetilde{F}_{\ell}
$$

where

$$
\widetilde{F}_{\ell}=\frac{\widetilde{D}_{\ell-1}^{\prime}+\left(m_{\ell}+\cdots+m_{k}-1\right) d_{\ell}^{\prime}+\widetilde{e}-\widetilde{e} / \widetilde{B}}{\widetilde{D}_{\ell-1}^{\prime}+\left(m_{\ell}+\cdots+m_{k}\right) d_{\ell}^{\prime}+\widetilde{e}} .
$$

and we have

$$
\frac{\partial \widetilde{F}_{\ell}}{\partial d_{\ell}^{\prime}}=\frac{-\widetilde{D}_{\ell}-\widetilde{e}+\left(m_{\ell}+\cdots+m_{k}\right) \widetilde{e} / \widetilde{B}}{\left(\widetilde{D}_{\ell-1}^{\prime}+\left(m_{\ell}+\cdots+m_{k}\right) d_{\ell}^{\prime}+\widetilde{e}\right)^{2}}<0
$$

where the negativity is derived from the fact $\widetilde{B} \geq m_{1}+\cdots+m_{k}$. By the exact same argument, we get the generalization of Theorem 30 :

Theorem 32. The Lojasiewicz exponent of $g=f_{1}^{m_{1}} \cdots f_{k}^{m_{k}}$ can be estimated as

$$
\theta_{0}(g) \leq 1-\frac{1}{\widetilde{B}}
$$

Furthermore, if $g$ has a non-exceptional Łojasiewicz monomial, the equality holds.

We comment that $\widetilde{B}=\max \left\{m_{1} b_{1 j}+\cdots+m_{k} b_{k j} \mid j=1, \ldots, n\right\}$.

\section{REFERENCES}

[1] O. M. Abderrahmane. The Eojasiewicz exponent for weighted homogeneous polynomial with isolated singularity. Glasg. Math. J. 59 (2017), 493-502.

[2] O. M. Abderrahmane. On the Eojasiewicz exponent and Newton polyhedron. Kodai Math. J. Vol. 28, (2005), 106-110.

[3] S. Brzostowski. The Eojasiewicz exponent of semiquasihomogeneous singularities. Bull. Lond. Math. Soc., 47 (2015), 848-852. 
[4] S. Brzostowski. A note on the Łojasiewicz exponent of non-degenerate isolated singularities, in Analytic and Algebraic Geometry 3, Łódź Univ. Press. (2019), 27-40.

[5] S. Brzostowski, T. Krasiński and G. Oleksik. A Conjecture on Eojasiewicz exponent J. of Singularties, 6 (2012), 124-130.

[6] C. Eyral and M. Oka, Non-compact Newton boundary and Whitney equisingularity for non-isolated singularities, Adv. Math. 316 (2017) 94-113.

[7] C. Eyral and M. Oka. Geometry of non-degenerate locally tame non-isolated singularities. arXiv:2005.01416v1, May, 2020.

[8] H. Hamm Lokale topologische Eigenschaften komplexer Räume, Math. Ann. 191, 1971, 235-252.

[9] A. G. Khovanskii Newton polyhedra and the genus of complete intersections Funkts. Anal. Prilozhen. 12, 1977, No.1 51-61.

[10] A. G. Kouchnirenko Polyèdres de Newton et Nombres de Milnor Inventiones Math. $32,1976,1-32$.

[11] K. Kurdyka. On gradients of functions definable in o-minimal structures, Ann. Inst. Fourier (Grenoble), 48, 1998, No. 3, 769-783.

[12] K. Kurdyka and A. Parusiński. w-stratification of subanalytic functions and the Eojasiewicz inequality, C. R. Acad. Sci. Paris Sér. I Math., 318, 1994, No. 2, 129 133.

[13] A. Lenarcik. On the Eojasiewicz exponent of the gradient of a holomorphic function, In: Singularities Symposium-Łojasiewicz 70, Banach Center Publ. 44, PWN, Warszaw 1998, 149-429.

[14] J. Milnor. Singular points of complex hypersurfaces, Annals of Math. Studies 61, Princeton Univ. Press, Princeton, N. J., Univ. Tokyo Press, Tokyo, 1968.

[15] T. Loi. On the global Eojasiewicz inequalities for the class of analytic logarithmicexponential functions, Ann. Inst. Fourier, Grenoble, 45-4 (1995), 951-971.

[16] S. Łojasiewicz,. Semi-analytic sets, in Global analysis and its applications (Lectures, Internat. Sem. Course, Internat. Centre Theoret. Phys., Trieste, 1972), Vol. III, 25-29, 1974.

[17] S. Łojasiewicz. Ensembles semi-analytiques, Inst. Hautes Études Sci., Bures-surYvette, 1965

[18] M. Oka. Non-degenerate complete intersection singularity. Hermann, Paris, 1997.

[19] M. Oka. Non-degenerate mixed functions. Kodai Math. J., 33 (2010), 1-62.

[20] M. Oka. Eojasiewicz exponents of non-degenerate holomorohic and mixed functions, Kodai Math. J., Vol. 41, 2018, 3, 620-651.

[21] G. Oleksik. The Eojasiewicz exponent of nondegenerate surface singularities, Acta Math. Hungar., 138 ( 2013), 179-199.

[22] G. Oleksik. The Lojasiewicz exponent of nondegenerate singularities. Univ. Iag. Acta Math. 47 (2009), 301-308.

[23] B. Tessier. Variétés polaires des singularités d'hypersurfaces. Invent. Math., 40(3) (1977), 267-292,

Department of Mathematics, Tokyo University of Science, Kagurazaka 1-3, SHINJUKU-KU, TOKYO 162-8601

Email address: oka@rs.tus.ac.jp 